\title{
Acute Dynamin Inhibition Dissects Synaptic Vesicle Recycling Pathways That Drive Spontaneous and Evoked Neurotransmission
}

\author{
ChiHye Chung, ${ }^{1}$ Barbara Barylko, ${ }^{2}$ Jeremy Leitz, ${ }^{1}$ Xinran Liu, ${ }^{1}$ and Ege T. Kavalali ${ }^{1,3}$ \\ Departments of ${ }^{1}$ Neuroscience, ${ }^{2}$ Pharmacology, and ${ }^{3}$ Physiology, University of Texas Southwestern Medical Center, Dallas, Texas 75390-9111
}

\begin{abstract}
Synapses maintain synchronous, asynchronous, and spontaneous forms of neurotransmission that are distinguished by their $\mathrm{Ca}^{2+}$ dependence and time course. Despite recent advances in our understanding of the mechanisms that underlie these three forms of release, it remains unclear whether they originate from the same vesicle population or arise from distinct vesicle pools with diverse propensities for release. Here, we used a reversible inhibitor of dynamin, dynasore, to dissect the vesicle pool dynamics underlying the three forms of neurotransmitter release in hippocampal GABAergic inhibitory synapses. In dynasore, evoked synchronous release and asynchronous neurotransmission detected after activity showed marked and unrecoverable depression within seconds. In contrast, spontaneous release remained intact after intense stimulation in dynasore or during prolonged $(\sim 1 \mathrm{~h})$ application of dynasore at rest, suggesting that separate recycling pathways maintain evoked and spontaneous synaptic vesicle trafficking. In addition, simultaneous imaging of spectrally separable styryl dyes revealed that, in a given synapse, vesicles that recycle spontaneously and in response to activity do not mix. These findings suggest that evoked synchronous and asynchronous release originate from the same vesicle pool that recycles rapidly in a dynamin-dependent manner, whereas a distinct vesicle pool sustains spontaneous release independent of dynamin activation. This result lends additional support to the notion that synapses harbor distinct vesicle populations with divergent release properties that maintain independent forms of neurotransmission.
\end{abstract}

\section{Introduction}

Synapses sustain three forms of neurotransmitter release with distinct $\mathrm{Ca}^{2+}$ dependence and time course (Neher and Sakaba, 2008). Presynaptic action potentials and ensuing $\mathrm{Ca}^{2+}$ influx cause either rapid (within a millisecond) synchronous vesicle fusion or asynchronous vesicle fusion loosely coupled to the timing of a presynaptic action potential (Lu and Trussell, 2000; Hefft and Jonas, 2005; Maximov and Südhof, 2005; Sun et al., 2007). In addition, neurotransmitter release can occur spontaneously in the absence of presynaptic action potentials albeit at a rate that can be modulated by intracellular $\mathrm{Ca}^{2+}$ levels (Fatt and Katz, 1952; Lou et al., 2005; Wasser and Kavalali, 2009; Xu et al., 2009). Despite recent advances in our understanding of the mechanisms that underlie the $\mathrm{Ca}^{2+}$ dependence of these three forms of release (Lou et al., 2005; Sun et al., 2007; Neher and Sakaba, 2008; Xu et al., 2009), the extent at which their maintenance relies on synaptic vesicle recycling remains unclear ( $\mathrm{Li}$ et al., 2005; Lin et al., 2005; Ertunc et al., 2007; Groemer and Klingauf, 2007). In addition, it remains uncertain whether these three forms of neurotransmis-

Received July 16, 2009; revised Nov. 17, 2009; accepted Nov. 28, 2009.

This work was supported by grants from the National Institute of Mental Health (E.T.K.). E.T.K. is an Established Investigator of the American Heart Association. We thank Drs. Joseph Albanesi and Mikhail Khvotchev for helpful discussions and for critically reading this manuscript.

Correspondence should be addressed to Dr. Ege T. Kavalali, Department of Neuroscience, University of Texas Southwestern Medical Center, 5323 Harry Hines Boulevard, Dallas, TX 75390-9111. E-mail: Ege.Kavalali@ UTSouthwestern.edu.

DOI:10.1523/JNEUROSCI.3427-09.2010

Copyright $\odot 2010$ the authors $\quad 0270-6474 / 10 / 301363-14 \$ 15.00 / 0$ sion originate from the same vesicle pool in excitatory (Sara et al., 2005; Groemer and Klingauf, 2007; Fredj and Burrone, 2009) or inhibitory (Mathew et al., 2008; Hablitz et al., 2009) synapses.

To address this question, we targeted dynamin GTPase, which is required to pinch synaptic vesicles from the plasma membrane during endocytosis (Praefcke and McMahon, 2004). The ability of dynamin to trigger vesicle scission requires GTP hydrolysis that can be reversibly inhibited by dynasore, a selective inhibitor of dynamin 1 and 2 (Macia et al., 2006; Kirchhausen et al., 2008). Previous work using synaptophysin-pHluorin-based monitoring of vesicle trafficking has shown that dynasore is a dosedependent inhibitor of synaptic vesicle endocytosis (Newton et al., 2006). This acute inhibition strategy provided two particular advantages in assessing the impact of dynamin-dependent vesicle endocytosis on neurotransmission. First, acute inhibition does not provide sufficient time for homeostatic compensation of dynamin function. For instance, such compensation has been reported for mouse knock-outs of P/Q-type voltage-gated calcium channels, which manifest a milder impairment of synaptic transmission compared with the one seen after acute inhibition of channel function (Piedras-Rentería et al., 2004). Second, reversible nature of dynasore-mediated dynamin inhibition allowed us to monitor the recovery kinetics of neurotransmission from inhibition.

Our results suggest that the maintenance of evoked synchronous or asynchronous release strictly depends on dynamin function at a rapid timescale, whereas spontaneous release does not 
require dynamin activation up to $1 \mathrm{~h}$. In contrast, spontaneous neurotransmission can be maintained after complete depletion of vesicle pools that sustain evoked neurotransmission. These findings imply that spontaneous recycling operates independent of evoked recycling machinery without a strict requirement for dynamin activation. In addition, we took advantage of the selectivity of dynasore action to dissect the spontaneous and evoked forms trafficking optically at individual synapses using spectrally separable styryl dyes. This analysis confirmed the premise that, in a given synapse, vesicles that recycle spontaneously and in response to activity do not mix.

\section{Materials and Methods}

Dissociated and organotypic slice culture. Dissociated hippocampal cultures were prepared from postnatal Sprague Dawley rats as described previously (Kavalali et al., 1999b). Unless stated otherwise, all experiments were performed after $14 \mathrm{~d}$ in vitro (DIV) when synapses reach maturity (Mozhayeva et al., 2002). For slice experiments, 400- $\mu$ m-thick transverse hippocampal slices were prepared from postnatal day $6-8$ mice pups expressing synaptophysin-pHluorin selectively in inhibitory synapses (spH64; gift from Dr. Venkatesh Murthy, Harvard University, Cambridge, MA) using a McIlwain tissue chopper and maintained in vitro on Millicell-CM filter inserts (Millipore Corporation). Culture medium was composed of minimum essential medium containing $20 \%$ equine serum (HyClone), $0.0012 \%$ ascorbic acid, $1 \mathrm{~mm}$ L-glutamine, 1 $\mathrm{mm} \mathrm{CaCl}, 2 \mathrm{~mm} \mathrm{MgSO}_{4} \cdot 7 \mathrm{H}_{2} \mathrm{O}, 12.87 \mathrm{~mm}$ dextrose, $5.25 \mathrm{~mm} \mathrm{NaHCO}_{3}$, $30 \mathrm{~mm}$ HEPES, and $0.17 \mathrm{~mm}$ insulin. Culture media were replaced after $24 \mathrm{~h}$ with media containing $1 \mathrm{~g} / \mathrm{ml}$ fluorodeoxyuridine (FUDR). After $24 \mathrm{~h}$ incubation in FUDR, culture media was replaced with media containing no FUDR and subsequently changed every $2 \mathrm{~d}$. Experiments were performed between 9 and $15 \mathrm{~d}$ in vitro.

Dynamin 1 GTPase activity assay. Dynamin 1 was expressed and purified from baculovirus-infected Sf9 cells following previously published procedures (Achiriloaie et al., 1999). GTPase activities of dynamin were measured by the release of ${ }^{32} \mathrm{Pi}$ from $\left[\mathrm{G}-{ }^{32} \mathrm{P}\right] \mathrm{GTP}$ by charcoal-based method (Higashijima et al., 1987) after incubation at room temperature for $15 \mathrm{~min}$ in a buffer containing $20 \mathrm{~mm}$ HEPES, pH 7.5, $0.1 \mathrm{M}$ $\mathrm{NaCl}, 4 \mathrm{~mm} \mathrm{MgCl}_{2}$, and $1 \mathrm{~mm}$ GTP. Phosphatidylinositol (4,5)bisphosphate $\left[\mathrm{PI}(4,5) \mathrm{P}_{2}\right]$-phosphatidylcholine (1:9 molar ratio) vesicles were prepared in $20 \mathrm{~mm}$ HEPES, pH 7.5, as described previously (Barylko et al., 1998).

Electron microscopy. For horseradish peroxidase (HRP) uptake experiments, hippocampal cultures were depolarized with $90 \mathrm{~mm} \mathrm{~K}{ }^{+}$containing Tyrode's solution, and, after removal of $90 \mathrm{mM} \mathrm{K}^{+}$solution, they were incubated with $25 \mathrm{mg} / \mathrm{ml} \mathrm{HRP}$ (Sigma) for $15 \mathrm{~min}$ in $1 \mu \mathrm{M}$ TTX, $10 \mu \mathrm{M}$ 6-cyano-7-nitroquinoxaline-2,3-dione (CNQX), and $50 \mu \mathrm{M}$ aminophosphonopentanoic acid (AP-5) with either $80 \mu \mathrm{M}$ dynasore or $0.2 \%$ DMSO. After HRP incubation, cells were washed twice with buffer and immediately fixed with $2 \%$ glutaraldehyde in $0.1 \mathrm{M}$ sodium phosphate buffer, $\mathrm{pH} 7.4$, at $37^{\circ} \mathrm{C}$ and washed with Tris- $\mathrm{Cl}(100 \mathrm{~mm}, \mathrm{pH} 7.4)$ buffer. Then, cells were incubated with $3,3^{\prime}$-diaminobenzidine $(0.1 \%$ DAB; Sigma) in Tris-Cl buffer and $\mathrm{H}_{2} \mathrm{O}_{2}(0.02 \%)$ for $15 \mathrm{~min}$ (DAB reaction) and washed with Tris- $\mathrm{Cl}$ buffer. At this point, cells were incubated in $1 \% \mathrm{OsO}_{4}$ for $30 \mathrm{~min}$ at room temperature and stained en bloc with $2 \%$ aqueous uranyl acetate for $15 \mathrm{~min}$, dehydrated in ethanol, and embedded in Poly/Bed 812, (Polysciences) for 24 h. Sections, $60 \mathrm{~nm}$ thick, were poststained with uranyl acetate and lead citrate. Images were obtained with a JEOL 1200 EX transmission microscope. All analyses were performed by an individual blind to the treatment conditions of the samples.

Electrophysiology. Pyramidal neurons were voltage clamped to -70 $\mathrm{mV}$ using an Axopatch 200B amplifier and Clampex 8.0 software (Molecular Devices), filtered at $2 \mathrm{kHz}$, and sampled at $5 \mathrm{kHz}$ at room temperature. The pipette solution contained the following (in $\mathrm{mm}$ ): 115 Cs- $\mathrm{MeSO}_{3}, 10 \mathrm{CsCl}, 5 \mathrm{NaCl}, 10 \mathrm{HEPES}, 0.6$ EGTA, 20 tetraethylammonium chloride, $4 \mathrm{Mg}$-ATP, $0.3 \mathrm{Na}_{2} \mathrm{GTP}$, and $10 \mathrm{QX}-314$ (lidocaine $\mathrm{N}$-ethyl bromide), pH 7.35 (300 mOsm) (Sigma). A modified Tyrode's solution used as the extracellular solution contained the following (in
mM): $150 \mathrm{NaCl}, 4 \mathrm{KCl}, 2 \mathrm{MgCl}_{2}, 10$ glucose, 10 HEPES, and $2 \mathrm{CaCl}_{2}, \mathrm{pH}$ $7.4(310 \mathrm{mOsm})$. High $\mathrm{K}^{+}$solutions contained equimolar substitution of $\mathrm{KCl}(90 \mathrm{~mm})$ for $\mathrm{NaCl}$. The recordings were not corrected for the liquid junction potential $(\sim 12 \mathrm{mV})$. Under our recording conditions, we estimate the equilibrium potential for $\mathrm{Cl}^{-}$at $-40 \mathrm{mV}$; therefore, we could record inward inhibitory current without altering our standard pipette solution. To record and isolate IPSCs, the ionotropic glutamate receptor antagonist CNQX (10 $\mu \mathrm{M}$; Sigma) and AP-5 (50 $\mu \mathrm{M}$; Sigma) were added to the bath solution. Spontaneous IPSCs were recorded in the same condition without field stimulation, and miniature IPSC (mIPSCs, or minis) were recorded in the addition of $1 \mu \mathrm{M}$ TTX. mIPSC events were analyzed manually to avoid false-positive and false-negative events. The detection threshold amplitude of mIPSC was $4 \mathrm{pA}$, and the SD of the baseline noise was $1.46 \pm 0.2 \mathrm{pA}$. The time period to search for local maximum was set to $8 \mathrm{~ms}$, time before a peak for a baseline was $4 \mathrm{~ms}$, and period to search a decay time was $8 \mathrm{~ms}$. We used a $1 \mathrm{~ms}$ period to average baseline. Dynasore (Sigma) was dissolved in DMSO under dark. To elicit evoked responses, electrical stimulation was delivered through parallel platinum electrodes in modified Tyrode's solution (duration, $1 \mathrm{~ms}$; amplitude, 20 $\mathrm{mA}$ ). The exchange between extracellular solutions was achieved by direct perfusion of solutions onto the field of interest by gravity.

Fluorescence imaging. The synaptophysin-pHluorin construct contained two pHluorins and was a generous gift from Drs. Y. Zhu and C. F. Stevens (The Salk Institute, La Jolla, CA). Hippocampal cultures were infected with lentivirus expressing the synaptophysin-pHluorin construct at $5 \mathrm{DIV}$, and imaging experiments were performed after $14 \mathrm{DIV}$ at room temperature. A modified Tyrode's solution containing $2 \mathrm{mM} \mathrm{Ca}^{2+}$, $10 \mu \mathrm{M}$ CNQX, and $50 \mu \mathrm{M}$ AP-5 was used in all experiments. Baseline images were obtained every second for $30 \mathrm{~s}$, and cultures were stimulated with field stimulation. After incubation with $80 \mu \mathrm{M}$ dynasore (Sigma) dissolved in DMSO (Sigma), stimulation was repeated on the same set of synaptic boutons.

For FM dye experiments, spontaneously recycling vesicles were labeled with FM dye for $10 \mathrm{~min}$ in the presence of $1 \mu \mathrm{M}$ TTX, $10 \mu \mathrm{M}$ CNQX, and $50 \mu \mathrm{M}$ AP-5. Activity-dependent recycling vesicle were labeled with FM dye for $90 \mathrm{~s}$ during $47 \mathrm{~mm} \mathrm{~K}^{+}$challenge in the presence of $10 \mu \mathrm{M} \mathrm{CNQX}$ and $50 \mu \mathrm{M}$ AP-5. After $10 \mathrm{~min}$ perfusion with dye-free solution, baseline images were obtained for $\sim 30 \mathrm{~s}$. Then, neurons were challenged with 90 $\mathrm{mM} \mathrm{K}^{+}$solution in the presence of $10 \mu \mathrm{M} \mathrm{CNQX}$ and $50 \mu \mathrm{M} \mathrm{AP}-5$ for $90 \mathrm{~s}$ for three times with $1 \mathrm{~min}$ wash in between. Images were obtained every second during three times of $90 \mathrm{~mm} \mathrm{~K}^{+}$challenges. Images were obtained by a cooled-intensified digital CCD camera (Roper Scientific) during illumination at $480 \pm 20 \mathrm{~nm}$ ( 505 dichroic long pass, $535 \pm 25$ bandpass) via an optical switch (Sutter Instruments). Images were acquired and analyzed using Metafluor Software (Universal Imaging Corporation).

For experiments on organotypic slice cultures, slices were perfused with a modified Tyrode's solution and stimulated by application of a Tyrode's solution containing $47 \mathrm{~mm} \mathrm{~K}^{+}$. Images were obtained using a Nikon D-Eclipse C1 confocal microscope and analyzed using EZ-C1 imaging software.

Spectral imaging experiments. Images were obtained by a Carl Zeiss LSM 510 META laser-scanning microscope equipped with LSM 510 Laser module (Carl Zeiss). To optimize image acquisition settings, we obtained spectral signature of each dye when nerve terminals were labeled with either dye alone or both dyes together.

For dual-labeling experiments, spontaneously recycling vesicles were labeled with either FM dye for $10 \mathrm{~min}$ in the presence of $1 \mu \mathrm{M}$ TTX, $10 \mu \mathrm{M}$ $\mathrm{CNQX}$, and $50 \mu \mathrm{M}$ AP-5. After 5 min perfusion with dye-free solution, neurons were incubated with the other FM dye during high $\mathrm{K}^{+}$challenge for $90 \mathrm{~s}$. Cells were subsequently excited with lasers at both 488 and $543 \mathrm{~nm}$, and the kinetics of fluorescence decrease for both FM dyes was simultaneously monitored ( $0.5 \mathrm{~Hz}$ acquisition rate) during $90 \mathrm{~mm} \mathrm{~K}^{+}$depolarization. The bleed-through of fluorescence emission from FM2-10 [ $N$-(3-triethylammoniumpropyl)-4-(4-(diethylamino)styryl)pyridinium dibromide] to red emission of FM5-95 [N-(3-trimethylammoniumpropyl)-4-(6-(4-(diethylamino)phenyl)hexatrienyl)pyridinium dibromide] was $\sim 15 \%$, whereas the bleed-through of FM5-95 emission to green was 3\%.

Data analysis. To quantify FM dye destaining kinetics, we selected $\sim 100$ synapses per experiment and normalized fluorescence values to the 
average baseline fluorescence at onset $[F(0)]$ without subtracting background fluorescence to avoid distortion in destaining profiles that may not reach a "true" baseline (Groemer and Klingauf, 2007). The time constants $\left(\tau_{\text {fast }}\right.$ and $\left.\tau_{\text {slow }}\right)$ were calculated by fitting the destaining profiles with a double-exponential function starting at stimulation onset. Twotailed unpaired $t$ test was used for all statistical comparisons, and values are given as mean \pm SEM.

\section{Results}

\section{Dynasore potently inhibits both the GTPase activity of dynamin 1 and the coupling between synaptic vesicle exocytosis and endocytosis}

To investigate the role of dynamin-dependent synaptic vesicle recycling in neurotransmission, we first tested whether the small molecule inhibitor dynasore suppresses GTPase activity of dynamin under our conditions. For this purpose, we used an in vitro GTPase activity assay that took advantage of the well characterized interaction between dynamin and $\mathrm{PI}(4,5) \mathrm{P}_{2}$. This interaction plays a critical role in driving synaptic vesicle endocytosis under physiological conditions (Cremona and De Camilli, 1997; Takei et al., 2005). In initial experiments, we confirmed that the GTPase activity of dynamin 1 is stimulated as a function of dynamin concentration in the presence of $\mathrm{PI}(4,5) \mathrm{P}_{2}$ (Higashijima et al., 1987; Barylko et al., 1998). Under our conditions ( $0.1 \mathrm{M} \mathrm{NaCl}$ and $0.24 \mu \mathrm{M}$ dynamin 1), GTPase activity was stimulated from a basal level of $0.9 \mathrm{~min}^{-1}$ in the absence of lipids, up to $86 \mathrm{~min}^{-1}$ in the presence of $4 \mu \mathrm{M} \mathrm{PI}(4,5) \mathrm{P}_{2}$. Dynasore inhibited $\mathrm{PI}(4,5) \mathrm{P}_{2}$ stimulated dynamin 1 activity in a dose-dependent manner, with $50 \%$ inhibition achieved at a dynasore concentration $\sim 40 \mu \mathrm{M}$ $(p<0.001)$ (Fig. 1A).

To examine whether dynasore is a functional inhibitor of endocytosis in central synapses, we used synaptophysin-pHluorininfected neurons and applied brief stimulation $(10 \mathrm{~Hz}$ for $10 \mathrm{~s}$ ) before and after dynasore application. Following the results of the in vitro assay, as well as a previous study (Newton et al., 2006), we opted to use $80 \mu \mathrm{M}$ dynasore for the experiments unless stated otherwise. As shown in Figure $1 B$, dynasore treatment inhibited the decay of pHluorin signal after the stimulation, suggesting that synaptic vesicle endocytosis was impaired (Newton et al., 2006). Moreover, we found an additional increase of fluorescence during the stimulation in the presence of dynasore, indicating inhibition of endocytosis during stimulation, which may impact short-term synaptic plasticity (Fig. $1 B$ ).

\section{Acute dynamin inhibition results in rapid synaptic depression and complete depletion of evoked neurotransmission}

To investigate the dependence of neurotransmission on dynaminmediated synaptic vesicle trafficking, we focused on inhibitory neurotransmission because several inhibitory central synapses display salient features of synchronous and asynchronous as well as spontaneous release ( $\mathrm{Lu}$ and Trussell, 2000; Hefft and Jonas, 2005; Maximov and Südhof, 2005). In these experiments, we stimulated hippocampal cultures at $10 \mathrm{~Hz}$ (as well as $20 \mathrm{~Hz}$; data not shown) either in the presence of $0.2 \%$ DMSO (control) or after 2-min-long incubation in 80 or $160 \mu \mathrm{M}$ dynasore (Fig. 1C). Dynasore treatment caused a marked depression of evoked IPSCs (eIPSCs) within $1 \mathrm{~s}$ after the onset of stimulation, indicating that the degree of synaptic depression at these frequencies is determined by dynamin-dependent vesicle reuse (Fig. 1D). These findings are consistent with previous observations that recycled vesicles contribute to neurotransmission within seconds (Delgado et al., 2000; Pyle et al., 2000; Sara et al., 2002; Ertunc et al., 2007).
After the $10 \mathrm{~Hz}$ trains of action potentials, we stimulated cells at 1 $\mathrm{Hz}$ for 2 min to monitor rapid recovery of depressed synaptic activity. Under control conditions, synaptic responses rapidly recovered $\sim 70 \%$ of their initial amplitude (Fig. $1 D$, open diamonds). In the presence of dynasore, however, synaptic responses failed to recover, indicating that the dynasore-mediated inhibition of endocytosis impairs synaptic vesicle resupply to the recycling synaptic vesicle pool (Fig. $1 D$, filled symbols). Threeminute-long dynasore treatment before stimulation did not cause a significant decrease in the amplitudes of initial eIPSCs $(2.6 \pm 0.2 \mathrm{nA}$ in control, $2.1 \pm 0.3 \mathrm{nA}$ in $80 \mu \mathrm{M}$ dynasore, and $2.4 \pm 0.3 \mathrm{nA}$ in $160 \mu \mathrm{M}$ dynasore; $n=7-9 ; p>0.3)$. This observation is consistent with the expectation that dynasore effect on release is use dependent and is not attributable to inhibition of exocytosis per se.

In the same set of experiments, we also monitored spontaneous mIPSCs before and after stimulation to examine their susceptibility to dynamin inhibition. Surprisingly, extensive and unrecoverable synaptic depression induced by stimulation in the presence of dynasore did not significantly alter spontaneous neurotransmission. When we compared mIPSCs before and after stimulation in dynasore, we only detected a small increase in the frequency of mIPSCs $(p<0.05$ in $80 \mu \mathrm{M}, p>0.3$ in $160 \mu \mathrm{M}$ dynasore) but no change in their amplitudes or other kinetic properties $(p>0.8)$ (Fig. $1 E-G)$. Thus, inhibition of dynamindependent synaptic recycling appears to impair action potentialdriven neurotransmission without altering the properties of spontaneous neurotransmission.

This interpretation relies on the assumption that spontaneous and evoked neurotransmission originate from the same set of synapses and activity-dependent depletion of vesicles giving rise to evoked release does not interfere with the maintenance of spontaneous release from individual synapses (Atasoy et al., 2008). To test this assumption, we took advantage of organotypic hippocampal cultures of spH64 transgenic mice, which express synaptophysin-pHluorin selectively in inhibitory presynaptic terminals (Li et al., 2005) (Fig. 2). To compare the rate of spontaneous and evoked release at individual synaptic boutons, we incubated slice cultures with folimycin $(80 \mathrm{nM})$, a high-affinity blocker of vacuolar ATPase (Dröse and Altendorf, 1997). Folimycin inhibits the refilling of neurotransmitters by trapping vesicles in an alkaline state, thus providing a cumulative measure of exocytosis (Sankaranarayanan and Ryan, 2001). In the absence of folimycin, we detected little change in fluorescence at rest, suggesting that spontaneous exocytosis and endocytosis are in balance under resting conditions (Fig. $2 A-C$ ). In folimycin, however, synaptophysin-pHluorin-labeled terminals showed a slow increase in fluorescence attributable to spontaneous release, consistent with a previous report in dissociated hippocampal cultures (Fig. 2A-C) (Atasoy et al., 2008). After monitoring the fluorescence for $10 \mathrm{~min}$ at rest, we stimulated the slice with bath application of $47 \mathrm{~mm} \mathrm{~K}^{+}$solution to visualize activity-dependent synaptic vesicle trafficking in all synaptic boutons within the field of view. When selected with respect to their responsiveness to the $47 \mathrm{mM} \mathrm{K}^{+}$challenge, the majority of synaptic boutons (615 of $890, \sim 70 \%$ ) that showed robust spontaneous release responded well to $47 \mathrm{~mm} \mathrm{~K}^{+}$application. The remaining population of synapses $(275$ of $890, \sim 30 \%$ ), which did not show any detectable spontaneous release in the presence of folimycin, nevertheless showed robust response to $47 \mathrm{mM} \mathrm{K}^{+}$stimulation. Overall, these data suggest that spontaneous fusion coexists with evoked release in the majority $(\sim 70 \%)$ of synapses in slices. This result is consistent with the findings of a similar analysis performed by our 
group on dissociated hippocampal cultures (Atasoy et al., 2008). Next, we optically analyzed how acute inhibition of dynamin in the presence of $80 \mu \mathrm{M}$ dynasore impacts spontaneous vesicle trafficking. In agreement with our electrophysiological observations, dynasore application did not cause a significant fluorescence increase at rest compared with controls, indicating that dynasore application does not alter the balance between exocytosis and endocytosis (Fig. 2A-C). On average, the rate of spontaneous fluorescence increase in the presence of folimycin was faster than the alterations in resting fluorescence levels in control or dynasore incubated slices $(p<0.05)$, whereas control and dynasore incubated slices did not show a significant difference in their fluorescence levels $(p>0.5)$ (Fig. 2D) (968 synapses tested). Together, our optical analysis in spH64 slice cultures suggests that acute inhibition of dynamin does not impair spontaneous synaptic vesicle recycling at individual synapses that show robust activity-dependent vesicle trafficking.

Extensive depletion of recycling vesicles after dynamin inhibition leaves spontaneous neurotransmission intact To ensure complete depletion of readily releasable pool vesicles, we used stronger high potassium stimulation to mobilize most of the recycling synaptic vesicle pool in dynasore and tested whether this maneuver was sufficient to impair both evoked and spontaneous neurotransmission (Fig. 3A). Two rounds of 30-s-long $90 \mathrm{mM} \mathrm{K}^{+}$application in the presence of dynasore ("depletion protocol") caused substantial suppression of neurotransmission as indicated by complete cessation of responses evoked at $20 \mathrm{~Hz}$ stimulation (Fig. $3 B, C$ ). The amplitude of the first eIPSC after $90 \mathrm{~mm} \mathrm{~K}^{+}$stimulation in the presence of dynasore application was only $5 \%$ of the amplitude of the eIPSC recorded before the stimulation, suggesting that nearly all vesicles within the readily releasable pool were depleted. In contrast, the same stimulation when applied in the presence of DMSO temporarily depressed eIPSCs down to $70 \%$ of their original amplitudes. Under the same conditions, $90 \mathrm{mM} \mathrm{K}^{+}$stimulation in the presence of dynasore caused only partial depletion of the vesicles released in response to hypertonic (+500 mOsm) sucrose stimulation. The amount of charge transfer induced by hypertonic sucrose solution after $90 \mathrm{~mm} \mathrm{~K}{ }^{+} /$dynasore application was $40 \%$ of the charge transferred
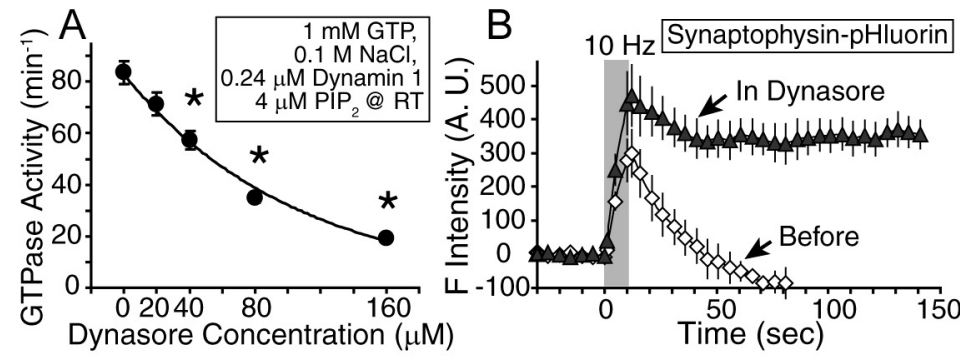

\section{C \\ Dynasore or DMSO

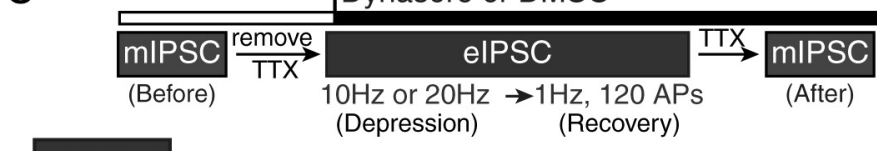

\section{elPSC}

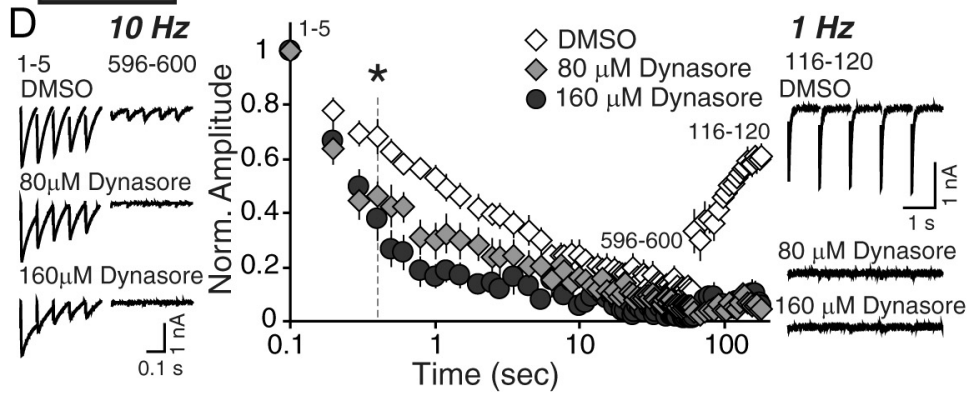

mIPSC
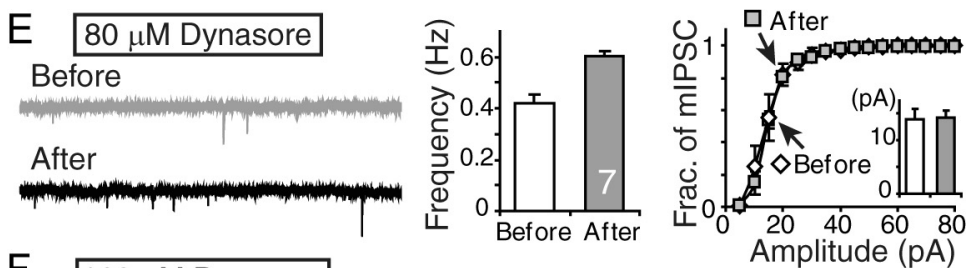

F $160 \mu \mathrm{M}$ Dynasore
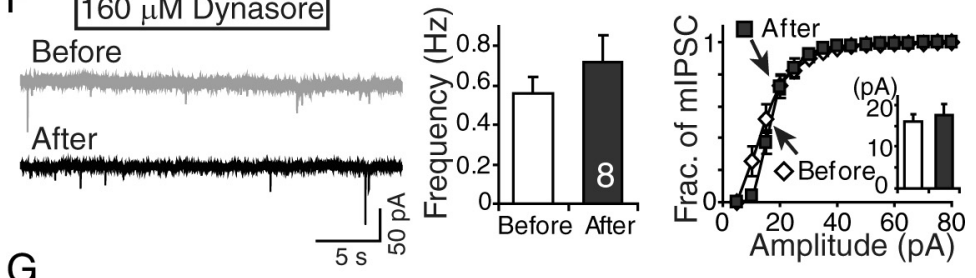

G

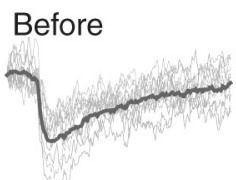

After

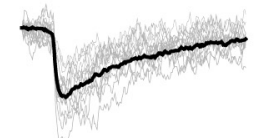

superimposed

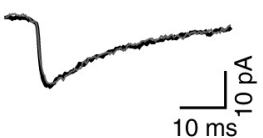

Figure 1. Endocytosis of synaptic vesicles during and after sustained stimulation requires GTPase activity of dynamin. $A$, Dynasore inhibits GTPase activity of $\mathrm{PI}(4,5) \mathrm{P}_{2}$-stimulated dynamin 1 in vitro in a concentration-dependent manner $(n=3-4) .{ }^{*} p<$ 0.05. B, Dynasore impairs endocytosis of synaptic vesicles during stimulation. The $10 \mathrm{~Hz}$ stimulation in the presence of $80 \mu \mathrm{m}$ dynasore showed little decay of pHluorin signal after stimulation as well as revealed additional rise of fluorescence during stimulation, suggesting that dynasore indeed impairs endocytosis in hippocampal synapses ( $n=450$ synapses from 4 coverslips). A.U., Arbitrary units. C, Experimental protocol. Cells were stimulated at $10 \mathrm{~Hz}$ for $60 \mathrm{~s}$ in the presence of either $0.2 \%$ DMSO (vehicle) or indicated concentrations of dynasore. mIPSCs were measured before and after transient incubation with DMSO or dynasore in the presence of TTX. AP, Action potentials. D, After application of dynasore, synaptic responses elicited by field stimulation (elPSCS) depressed faster during successive stimulation at $10 \mathrm{~Hz}$ for $1 \mathrm{~min}$, leading to temporary depletion of available synaptic vesicles at terminals. In addition, dynasore at the concentrations we used ( 80 and $160 \mu \mathrm{m}$ ) blocked the recovery of synaptic responses after depression $\left(1 \mathrm{~Hz}\right.$; right), ${ }^{*} p<0.05 . E, F, 0$ nly the increase in the frequency of mIPSCs before and after $10 \mathrm{~Hz}$ stimulation in $80 \mu \mathrm{m}$ dynasore was statistically significant $(p<0.05)$. The frequency of mIPSC $\operatorname{in} 160 \mu \mathrm{m}$ dynasore $(p>0.3)$ and the amplitude of mIPSCs in both concentrations of dynasore remained unchanged $(p>0.9)$. G, Averaged unitary responses before and after $80 \mu$ m dynasore application can be fully superimposed, suggesting that dynasore treatment does not alter the kinetics of individual mIPSCs. 

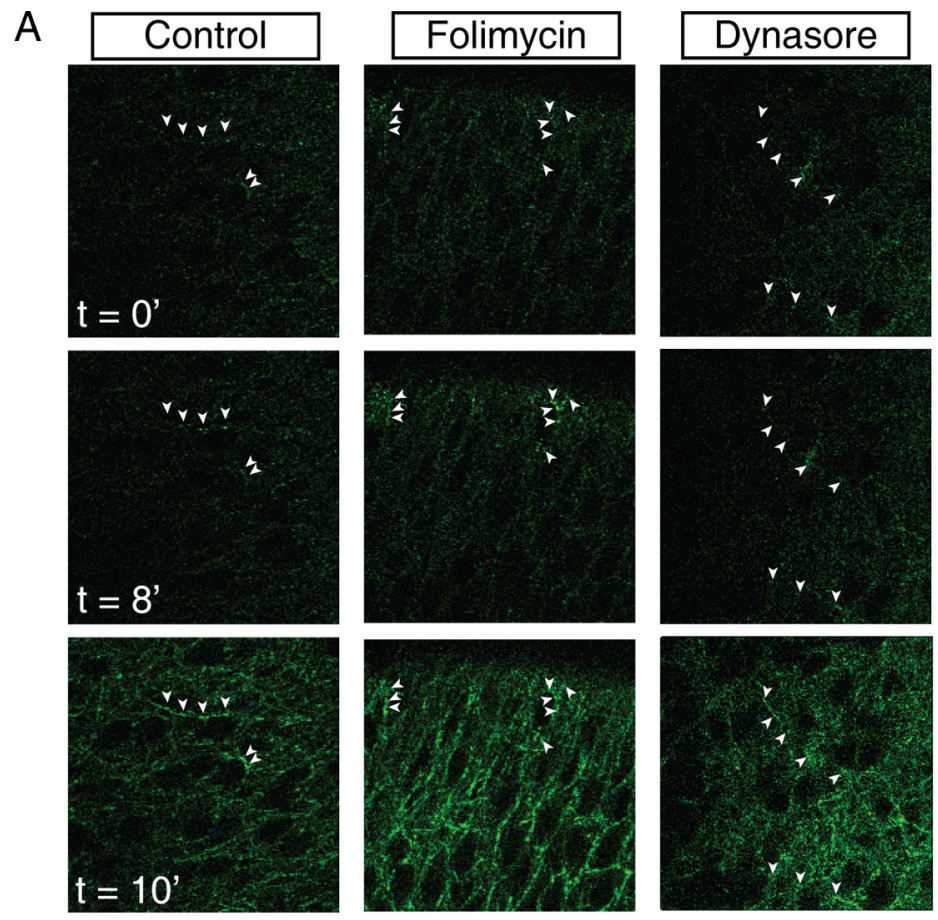

B Spontaneous $47 \mathrm{mM} \mathrm{K}^{+}$
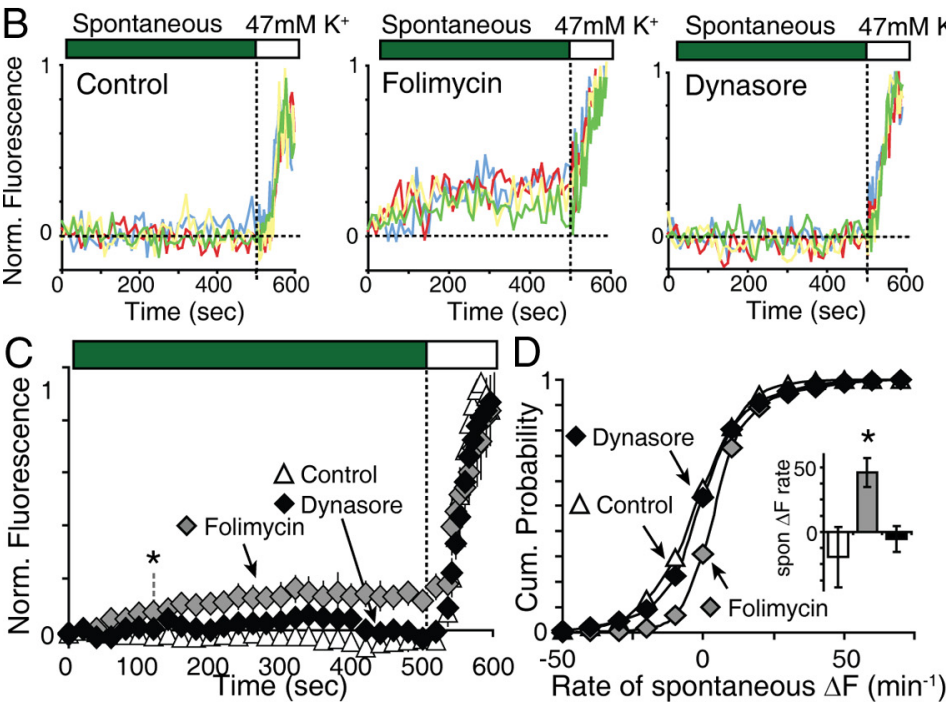

Figure 2. Spontaneous and evoked transmission occur at the same synapses and are differentially affected by dynasore. $\boldsymbol{A}$, Top, Sample images of spH64 slice cultures of control (left), in $80 \mathrm{~nm}$ folimycin (middle), and in $80 \mu \mathrm{m}$ dynasore (right). The white arrows point to the puncta that show markedly visible increases in fluorescence during folimycin application. Middle, Same sample images after 10 min incubation depicting the changes in fluorescence at rest presumably attributable to spontaneous release (Atasoy et al. 2008). The presence of folimycin caused a slight increase of fluorescence with a relatively constant rate over $10 \mathrm{~min}$. In contrast, the presence of dynasore did not trigger a comparable change in resting fluorescence levels, suggesting that spontaneous synaptic vesicle recycling was not affected by dynamin inhibition. Bottom, Robust increase in fluorescence was observed after application of $47 \mathrm{~mm} \mathrm{~K}^{+}$solution in all cases. $\boldsymbol{B}$, Sample traces depict the fluorescence change at the level of individual synapses. Note that folimycin incubation (middle) alters the rate of fluorescence increase at rest and during depolarization at each selected synaptic

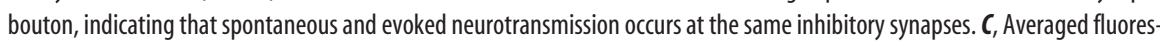
cence changes at rest and during elevated $\mathrm{K}^{+}$application under each condition ( $n=934,890,968$ synapses from 6 slices each for control, folimycin, dynasore, respectively). Fluorescence values were normalized with respect to their highest respective levels during $47 \mathrm{~mm} \mathrm{~K}^{+}$application. Note that fluorescence changes at rest in the presence of dynasore were not different from control $(p>0.5)$, whereas notable increases were detected in the presence of folimycin $\left({ }^{*} p<0.05\right)$ suggesting that application of dynasore hardly interferes with spontaneous synaptic vesicle recycling. $\boldsymbol{D}$, Cumulative probability histogram of the rate of spontaneous fluorescence increase in individual synapses. The inset shows the average rate of spontaneous fluorescence increase $(\Delta F / \mathrm{min})$ under each condition. The spontaneous fluorescence increase rate $(\Delta F / \mathrm{min})$ in folimycin was faster than in dynasore or in control conditions, although no significant difference was observed between control and dynasore group $\left({ }^{*} p<0.05\right)$. in the presence of DMSO (Fig. $3 B-D$ ) $(p<0.001)$, suggesting that a significant number of synaptic vesicles respond to hypertonicity but not to action potentials. This finding agrees with the previous reports that hypertonicity-driven release is partly mediated by a reluctant synaptic vesicle pool that is not mobilized during activity (Moulder and Mennerick, 2005; Moulder et al., 2007). These reluctant vesicles may instead contribute to spontaneous neurotransmission.

If the spontaneous and evoked forms of release originated from the same population of vesicles, we would expect to see equal suppression and similar time courses of recovery for evoked and spontaneous forms of release after dynamin inhibition. However, in contrast to substantial inhibition of action potential evoked release and the marked reduction of hypertonicity-driven neurotransmission, $90 \mathrm{mM} \mathrm{K}^{+}$stimulation during dynamin inhibition did not reduce spontaneous neurotransmission below its baseline level (Fig. 3I-K). In these experiments, after temporary depletion by two consecutive applications of $90 \mathrm{~mm}$ $\mathrm{K}^{+}$solution in the presence of dynasore, we removed dynasore from the extracellular solution and monitored the time course of recovery for the two forms neurotransmission (Fig. 3E). Removal of dynasore after $90 \mathrm{mM} \mathrm{K}^{+}$stimulation resulted in extremely slow recovery of evoked responses (Fig. $3 F-H$ ). eIPSCs recovered only $15 \%$ of their initial amplitudes $10 \mathrm{~min}$ after perfusion of dynasorefree solution, and on average $50 \%$ recovery was reached at $185 \mathrm{~s}$ (Fig. 3G,H). In contrast, the frequency and the amplitudes of spontaneous sIPSCs, monitored in the absence of TTX for a direct comparison with the recovery of eIPSCs, were unaltered throughout this period (Fig. $3 K$ ).

Here, it is important to note that, in dissociated hippocampal cultures, inhibition of excitatory neurotransmission abolishes network activity seen under normal conditions (Virmani et al., 2006). Therefore, in a typical experiment, inclusion of TTX does not cause a robust difference between the frequencies of sIPSCs and mIPSCs (unlike sEPSCs and mEPSCs). We also replicated these findings using moderate stimulation $(10 \mathrm{~Hz}$ for $60 \mathrm{~s})$ in the presence of 80 or $160 \mu \mathrm{M}$ dynasore. In this case, after removal of dynasore, evoked IPSCs slowly recovered 43 or 13\% of their original amplitudes (in 80 or 160 $\mu \mathrm{M}$ dynasore, respectively). We detected $80 \%$ recovery by $320 \mathrm{~s}$ after $80 \mu \mathrm{M}$ dynasore-mediated inhibition, whereas 
A

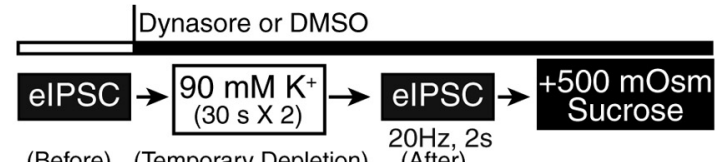

eIPSC Sucrose

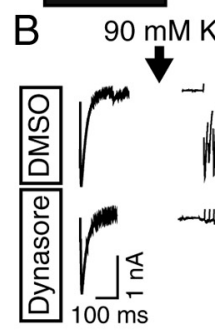

$2 \mathrm{~Hz}$
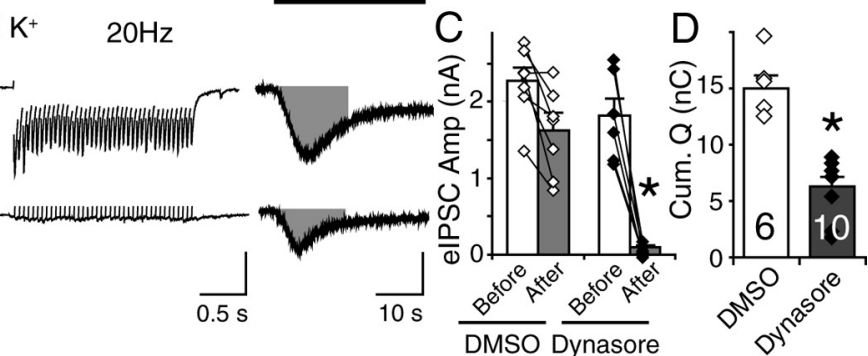

E

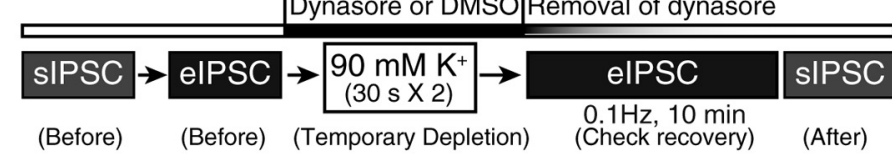

\section{eIPSC}
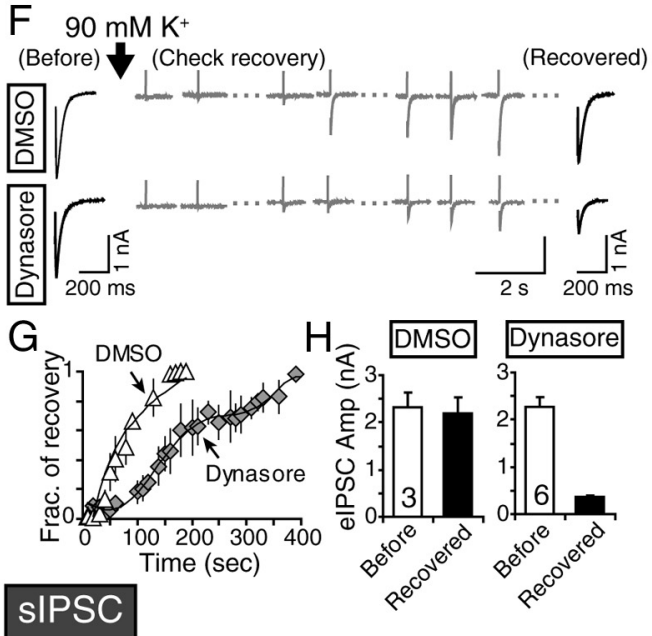

I After $90 \mathrm{mM} \mathrm{K}^{+}+80 \mu \mathrm{M}$ Dynasore removal

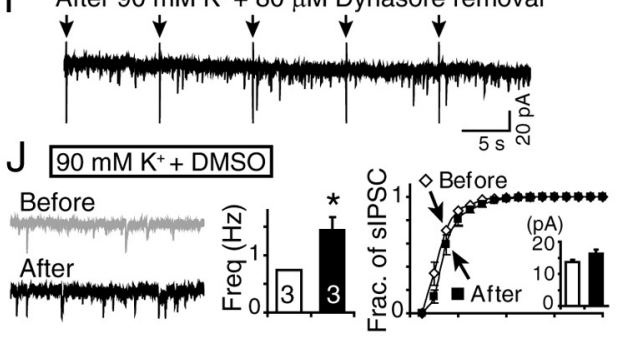

$\mathrm{K} 90 \mathrm{mM} \mathrm{K}^{+}+$Dynasore

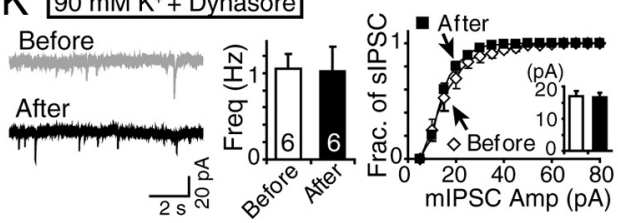

Figure 3. Extensive depletion of recycling vesicles after dynamin inhibition leaves spontaneous neurotransmission intact. $\boldsymbol{A}$, Experimental protocol using $90 \mathrm{~mm} \mathrm{~K}$ /dynasore application to deplete recycling synaptic vesicles. $\boldsymbol{B}$, Repeated $90 \mathrm{~mm} \mathrm{~K}{ }^{+}$/ dynasore application completely suppressed subsequent evoked synaptic responses and inhibited hypertonic sucrose-induced release by $60 \%$. C, The amplitudes of elPSC before and after $90 \mathrm{~mm} \mathrm{~K}^{+}$application in the presence of DMSO were not significantly different $(n=7 ; p>0.1)$. However, elPSC amplitudes after $90 \mathrm{~mm} \mathrm{~K}^{+} /$dynasore were decreased by $95 \%$, suggesting that $90 \mathrm{~mm}$ $\mathrm{K}^{+} /$dynasore application depleted available vesicles that fuse in response to action potentials $(n=6 ; p<0.001)$. $\boldsymbol{D}$, The charge transfer induced by sucrose application (15s) in the presence of dynasore was $40 \%$ of control ( $n=6-10 ; p<0.001)$, suggesting
sIPSCs were unaffected during and after the extensive suppression of evoked neurotransmission.

Acute inhibition of dynamin activity during depolarization decreases total synaptic vesicle number but leaves spontaneous synaptic vesicle trafficking intact

To examine whether strong high $\mathrm{K}^{+}$stimulation in the presence of dynasore causes detectable changes in synaptic vesicle numbers at the ultrastructural level, we analyzed morphology of synaptic terminals after depletion protocol using electron microscopy. In the first set of experiments, cells were challenged with $90 \mathrm{~mm} \mathrm{~K} \mathrm{~K}^{+}$ stimulation two consecutive times $(30 \mathrm{~s}$ each) in the presence of either $0.1 \%$ DMSO or $80 \mu \mathrm{M}$ dynasore before fixation (Fig. $4 A-C$ ). Figure 4 summarizes the results of electron micrograph analysis by an observer blind to the treatment conditions. This analysis revealed that incubation with dynasore during strong activity depletes a substantial number of synaptic vesicles despite the large size of the resting pool (Fig. 4C). This result suggests that synaptic vesicle reuse during strong depolarization requires dynamin activation. This significant but modest decrease in synaptic vesicle numbers is consistent with the notion that these boutons contain a large non-releasable resting pool

$\leftarrow$

that hypertonicity mobilizes a larger vesicle pool compared with $20 \mathrm{~Hz}$ stimulation. $\boldsymbol{E}$, Experimental protocol to monitor repopulation kinetics of synaptic vesicles after temporary depletion. After $90 \mathrm{~mm} \mathrm{~K}^{+} /$dynasore application, recovery of synaptic activity was monitored during $0.1 \mathrm{~Hz}$ stimulation in the absence of dynasore. $F$, Sample recordings depicting recovery of elPSCs after $90 \mathrm{~mm} \mathrm{~K}^{+}$stimulation (with or without dynasore). G, Recovery of synaptic responses after $90 \mathrm{~mm} \mathrm{~K}^{+}$ stimulation (with or without dynasore) is plotted as a function of time. The time courses of recovery are normalized with respect to the amplitude of final elPSC after $10 \mathrm{~min}$ of perfusion. $\boldsymbol{H}$, Bar graphs depict the amplitudes of elPSCS before application of $90 \mathrm{~mm} \mathrm{~K}^{+}$with either DMSO or dynasore and after 10 min wash of $90 \mathrm{~mm} \mathrm{~K}^{+} / \mathrm{DMSO}$ or $90 \mathrm{~mm} \mathrm{~K}^{+}$/dynasore. I, A sample recording after temporary depletion induced by two executive applications of $90 \mathrm{~mm} \mathrm{~K}{ }^{+}$in the presence of dynasore. Arrows indicate the time points when stimulations were applied. Note that, under these conditions, spontaneous neurotransmission remains intact, whereas elPSCs are abolished. $\boldsymbol{J}, \boldsymbol{K}$, The levels of sIPSCs (spontaneous transmission measured in the absence of TTX) before and after application of $90 \mathrm{mM}$ $\mathrm{K}^{+} / 0.2 \% \mathrm{DMS} 0$ or $90 \mathrm{~mm} \mathrm{~K}^{+} / 80 \mu \mathrm{m}$ dynasore were analyzed. The frequency of sIPSCs was not decreased after $90 \mathrm{~mm} \mathrm{~K}^{+} /$ dynasore $(n=6 ; p>0.9)$, suggesting differential regulation of synaptic vesicles for action potential-driven release and spontaneous release $(n=3-6)$. The amplitude of $s$ IPSC $s$ was not altered $(n=6 ; p>0.6)$. Challenge with $90 \mathrm{~mm} \mathrm{~K}^{+}$with DMSO increased the frequency $(n=3 ; p<0.01)$ but not the amplitude $(n=3 ; p>0.6)$ of sIPSCs. ${ }^{*} p<0.05$. 


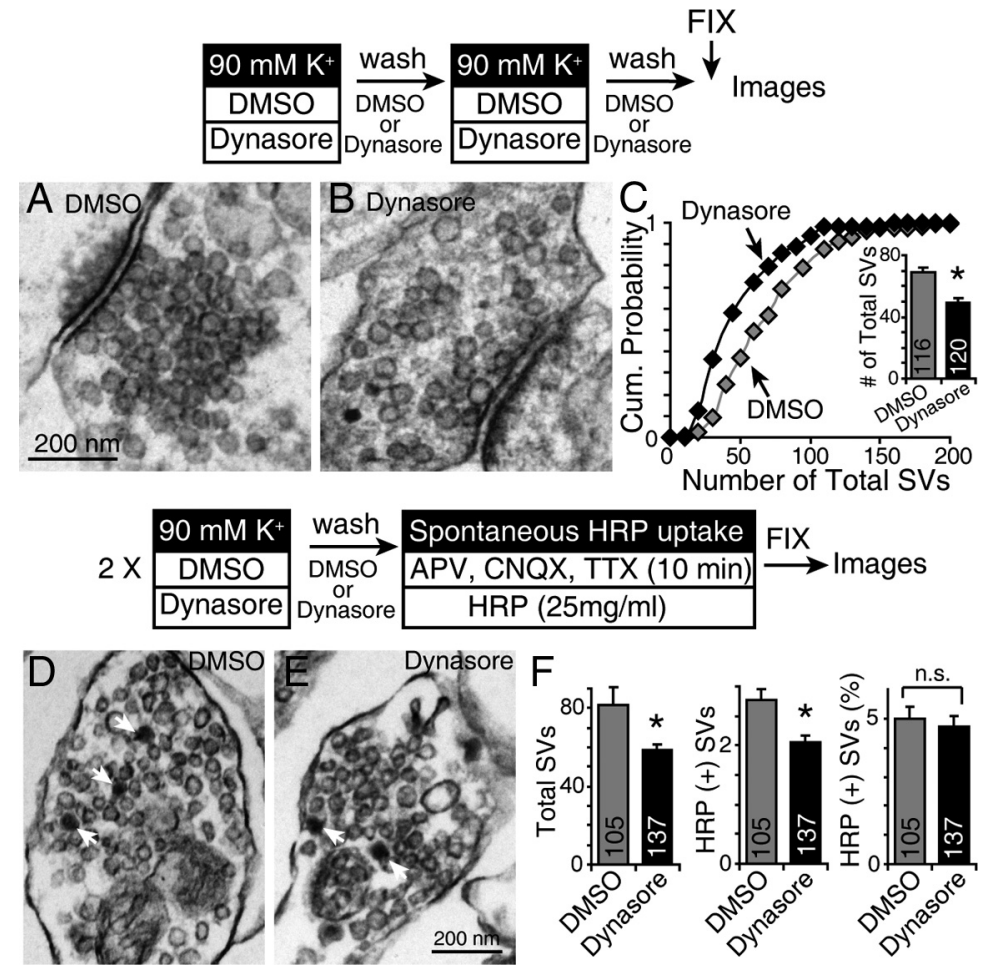

Figure 4. Incubation with dynasore during stimulation causes vesicle depletion. $A-C$, Before fixation and visualization, cells were challenged with $90 \mathrm{~mm} \mathrm{~K}^{+}$solution ( $2 \times$ for $30 \mathrm{~s}$ each) in the presence of either $0.1 \%$ DMSO or $80 \mu \mathrm{m}$ dynasore. $\boldsymbol{A}, \boldsymbol{B}$, Representative electron micrographs of synaptic terminals after $90 \mathrm{~mm} \mathrm{~K}^{+} / D M S 0$ or $90 \mathrm{~mm} \mathrm{~K}^{+} /$dynasore. C, Application of $90 \mathrm{~mm}$ $\mathrm{K}^{+} /$dynasore solution decreased the number of total synaptic vesicles (Total SVs) $(p<0.001)$. $\boldsymbol{D}-\boldsymbol{F}$, Cells were incubated with HRP ( $25 \mathrm{mg} / \mathrm{ml})$ for $15 \mathrm{~min}$ in the presence of TTX after depletion protocol to assess the degree of spontaneous synaptic vesicle trafficking. $\boldsymbol{D}, \boldsymbol{E}$, Representative electron micrographs of synaptic terminals. White arrows indicate synaptic vesicles that took up HRP under resting conditions after $90 \mathrm{~mm} \mathrm{~K}^{+} /$dynasore-induced depletion. $\boldsymbol{F}$, The $90 \mathrm{~mm} \mathrm{~K}^{+} /$dynasore-induced depletion decreased the number of the total synaptic vesicles $(p<0.001)$. The number of HRP-positive synaptic vesicles was slightly decreased in the dynasore group $(p<0.01)$. However, the relative number of HRP-positive synaptic vesicles per synapse remains unchanged $(p>0.6) .{ }^{*} p<0.05$

(Harata et al., 2001), which may contribute to spontaneous release (Fredj and Burrone, 2009).

To investigate whether this strong synaptic vesicle depletion protocol affects spontaneous synaptic vesicle trafficking, we took advantage of HRP to selectively label recycling vesicles (Fig. $4 D-$ $F)$. To label spontaneously recycling vesicles after activitydependent vesicle depletion, we incubated cells with HRP (25 $\mathrm{mg} / \mathrm{ml}$ ) for $15 \mathrm{~min}$ in the presence of TTX at the end of the high $\mathrm{K}^{+}$-induced depletion protocol. Figure 4, $D$ and $E$, shows sample electron micrographs of synaptic terminals after $90 \mathrm{~mm} \mathrm{~K}^{+}$application in either DMSO or dynasore. White arrows indicate spontaneously recycling synaptic vesicles that took up HRP after $90 \mathrm{~mm} \mathrm{~K}^{+}$depletion protocol. Under these conditions, we again observed a reduction in the total number of synaptic vesicles after activity-dependent vesicle depletion $(81.85 \pm 8.8$ for DMSO, $58.44 \pm 3.14$ for dynasore; $p<0.001$ ) (Fig. $4 F$, left), which was also reflected in a slight decrease in the number of HRP-positive synaptic vesicles (Fig. $4 F$, middle). This small reduction in spontaneous HRP uptake can be caused by dynasoremediated suppression of asynchronous release (see next section). Nevertheless, given the decrease in the total number of synaptic vesicles, the fraction of HRP-positive synaptic vesicles in a given synapse was not altered in the presence of dynasore (Fig. $4 F$, right). Under all conditions, the fraction of spontaneously recycling vesicles detected with HRP was small ( $~ 5 \%)$, which may be partly attributable to the low efficacy of spontaneous recycling as well as HRP labeling. This finding suggests that, after $90 \mathrm{~mm} \mathrm{~K}{ }^{+}$-mediated depletion of recycling synaptic vesicles, spontaneous uptake of HRP remains relatively intact, consistent with the premise that at individual synapses spontaneous synaptic vesicle recycling is relatively unaffected by dynamin inhibition and vesicle depletion.

\section{Dynamin dependence of asynchronous neurotransmitter release}

In the preceding set of experiments, removal of $90 \mathrm{mM} \mathrm{K}^{+}$stimulation under control conditions resulted in nearly twofold increase in spontaneous mIPSC activity (Fig. $3 J$ ). This increase in spontaneous transmission could be attributed to asynchronous release typically observed after extensive stimulation ( $\mathrm{Lu}$ and Trussell, 2000; Otsu and Murphy, 2004; Otsu et al., 2004; Maximov and Südhof, 2005). Interestingly, coapplication of dynasore and $90 \mathrm{mM} \mathrm{K}^{+}$solution completely inhibited this increase in neurotransmission (Fig. 3K). Therefore, next we investigated whether asynchronous neurotransmitter release also depended on dynamin-dependent synaptic vesicle recycling. To address this question, we applied a round of $10 \mathrm{~Hz}$ stimulation for $5 \mathrm{~s}$ in $8 \mathrm{mM} \mathrm{Ca}^{2+}$ to exacerbate the extent of asynchronous release, followed by a second round of $10 \mathrm{~Hz}$ stimulation in the presence of $80 \mu \mathrm{M}$ dynasore (Fig. $5 A$ ). The amount of asynchronous neurotransmitter release was measured as the charge transfer (Fig. 5D, shaded area) after the cessation of stimulation (Maximov and Südhof, 2005).

Under these conditions, dynasore application did not only cause faster depression of evoked release during stimulation (Fig. $5 C$, right) but also a marked reduction in the amount of asynchronous release detected at the end of the stimulation train (Fig. $5 B, D)$. The amplitudes of first eIPSCs were comparable in the presence of DMSO or dynasore, suggesting that exocytosis was not altered by dynamin inhibition (Fig. 5C, left). However, the decay time course of the last eIPSC in the train was significantly faster in the presence of dynasore, indicating loss of asynchronous release that follows the burst of action potentials (Fig. $5 E$ ). These results suggest that synaptic vesicles recycled in a dynamindependent manner drive asynchronous release that follows extensive activity. This finding agrees with the previous proposal that synchronous and asynchronous forms of neurotransmission originate from the same pool of vesicles albeit with distinct $\mathrm{Ca}^{2+}$ dependence (Otsu and Murphy, 2004; Otsu et al., 2004; Sun et al., 2007).

\section{Maintenance of spontaneous neurotransmission does not require the GTPase activity of dynamin}

Results presented so far are consistent with the hypothesis that synchronous as well as asynchronous forms of neurotransmitter release originate from the same population of vesicles that rapidly recycle in a dynamin-dependent manner. In contrast, spontane- 
ous neurotransmission appears to originate from a distinct vesicle pool that maintains its activity even after complete suppression of other forms of release. However, these findings do not exclude the possibility that spontaneous neurotransmission operates in a dynamindependent manner albeit at slower timescale. To test this premise, we monitored spontaneous neurotransmission up to $1 \mathrm{~h}$ and examined whether sustained treatment with dynasore at rest (in the presence of TTX) altered the properties of mIPSCs as well as subsequent eIPSCs. In these experiments, we initially measured the amplitudes of eIPSCs followed by application of TTX. Once a stable baseline for mIPSCs was obtained $(\sim 3 \mathrm{~min}$ in DMSO), we applied $80 \mu \mathrm{M}$ dynasore and monitored mIPSCs for $1 \mathrm{~h}$. At the end of the $1 \mathrm{~h}$ recording period, we cleared TTX from the extracellular medium $(\sim 5 \mathrm{~min})$ in the presence of $80 \mu \mathrm{M}$ dynasore and restimulated eIPSCs (Fig. 6A). As shown in Figure 6, we detected a significant increase in the frequency of mIPSCs $(p<$ 0.001) (Fig. 6 $B, C$ ). During this period, amplitudes of mIPSC also showed an increasing trend that did not reach significance $(p=0.056)$ (Fig. $6 D)$, which is consistent with a postsynaptic effect of dynasore. These data show that spontaneous neurotransmission does not rely on dynamin-dependent synaptic vesicle recycling up to $1 \mathrm{~h}$. One hour treatment with dynasore at rest caused up to $\sim 60 \%$ reduction in the amplitudes of subsequent eIPSCs (Fig. 6E), indicating that dynasore is still capable of inhibiting trafficking at rest, although this inhibition spares spontaneous neurotransmission. Interestingly, the remaining amount of evoked release is comparable with the level of eIPSCs detected in mice deficient in dynamin 1 (Ferguson et al., 2007). Together, these observations are consistent with the possibility that vesicle pools maintaining spontaneous and evoked neurotransmission mix with a slow rate in the order of hours (Sara et al., 2005).

Simultaneous measurement of the release kinetics of vesicles recycled spontaneously or during stimulation using spectrally separable styryl dyes

Our results so far strongly suggest that spontaneous and evoked forms of neurotransmission originate from two vesicle pools that can be distinguished by their reliance of dynamin function. This premise is consistent with a previous report from our laboratory that suggested that spontaneous and activity-dependent vesicle recycling originate from distinct pools with limited crosstalk (Sara et al., 2005). In contrast, a recent study monitored the release of vesicles recycled spontaneously or during stimulation using spectrally separable styryl dyes and suggested that the two forms of release originate from the same set of vesicles that recycle during activity (Groemer and Klingauf, 2007). In the next set of

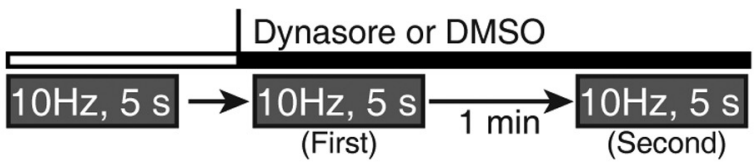

$\mathrm{B}$ in $8 \mathrm{mM} \mathrm{Ca}^{2+}+\mathrm{DMSO}$

C
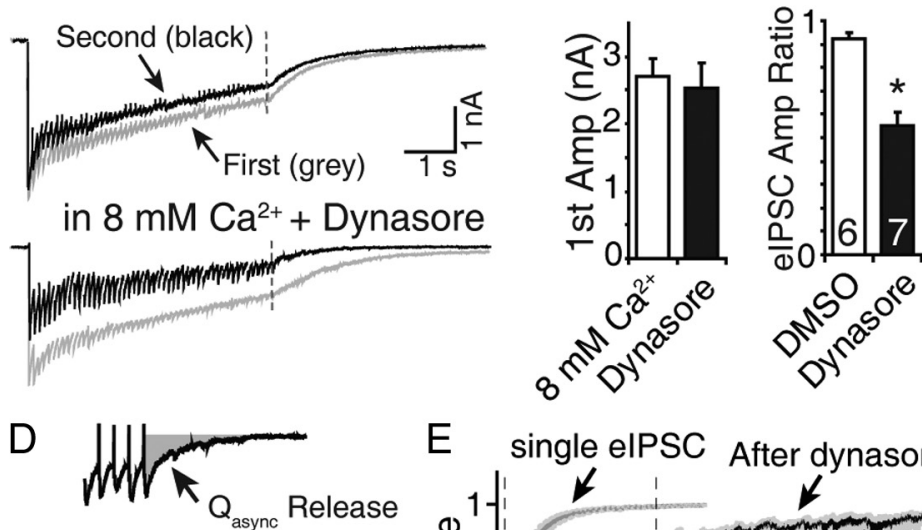

E single eIPSC After dynasore

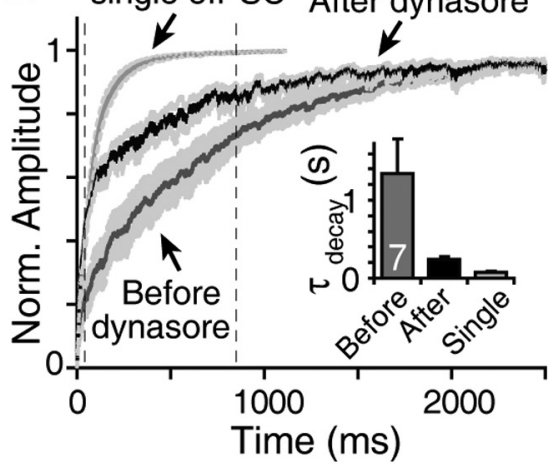

Figure 5. Dynamin dependence of asynchronous neurotransmitter release. $\boldsymbol{A}$, Experimental protocol. Cells were stimulated at a frequency of $10 \mathrm{~Hz}$ for 50 action potentials. Then, after 2-min-long application of dynasore (or DMSO control), cells were $(n=7-13 ; p>0.8)$. However, subsequent stimulation caused a decrease in the initial eIPSC amplitudes in the presence of 作 0.001 ) of the first round. ${ }^{*} p<0.05$. D. Total asynchronous release was quantified as the area from the onset of final stimulation to the baseline $\left(Q_{\text {async }}\right.$ release, gray area). $Q_{\text {async }}$ release was decreased in the presence of dynasore $(n=7 ; p<0.001)$ but not in DMSO $(n=6, p>0.5)$. First, $Q_{\text {async }}$ release in dynasore was not different from in $8 \mathrm{~mm} \mathrm{Ca}{ }^{2+}$ before dynasore application $(p>$ .4). ${ }^{*} p<0.05$. E, Decay phase of the last (50th) elPSC was normalized and fitted with a single-exponential function to extract the time constant $\tau_{\text {decay }} . \tau_{\text {decay }}$ of the 50th elPSC before dynasore application was $1242 \pm 420 \mathrm{~ms}$, and after dynasore application, it was reduced to $237 \pm 38 \mathrm{~ms}$ ( $n=7 ; p<0.05$ between the dotted time line). $\tau_{\text {decay }}$ of single elPSCs were measured as $78 \pm 7 \mathrm{~ms}$.

experiments, we aimed to gain new insight into this discrepancy by taking advantage of the differential dynamin dependence of the two forms of synaptic vesicle trafficking. For this purpose, initially we revisited previous results and labeled hippocampal synapses with spontaneous uptake of FM dyes FM2-10 $(400 \mu \mathrm{M})$ or FM1-43 [ $N$-(3-triethylammoniumpropyl)-4-(4-(dibutylamino)styryl) pyridinium dibromide] $(8 \mu \mathrm{M})$ in the presence of a Tyrode's solution containing activity blockers (CNQX, AP-5, and TTX) for $10 \mathrm{~min}$. After removal of excess dye ( $\sim 10 \mathrm{~min})$, synapses were challenged with $90 \mathrm{mM} \mathrm{K}^{+}$solution repeatedly to mobilize all releasable synaptic vesicles. After $10 \mathrm{~min}$ rest, the same set of synapses were loaded with FM2-10 or FM1-43 in depolarizing $47 \mathrm{mM} \mathrm{K}^{+}$solution to induce activity-dependent dye uptake and subsequently challenged with a $90 \mathrm{~mm} \mathrm{~K}^{+}$containing solution repeatedly to trigger dye release (Fig. 7). Under these conditions, synapses spontaneously labeled with FM2-10 showed significantly slower destaining during $90 \mathrm{mM} \mathrm{K}^{+}$stimulation, in agreement with our previous report (Fig. 7 A, C) (Sara et 


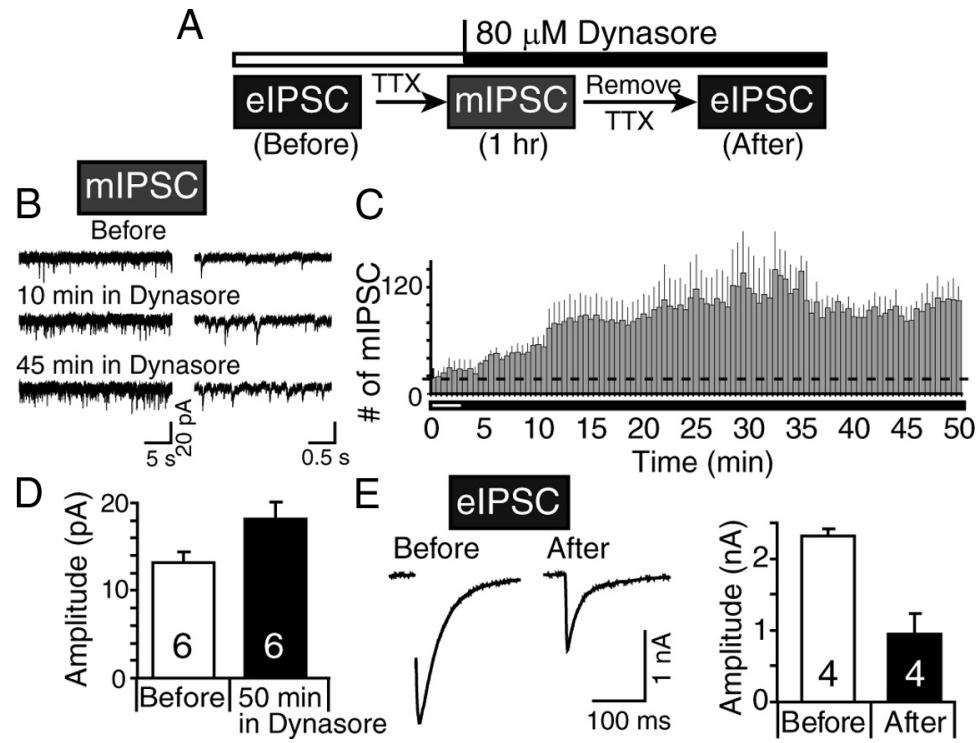

Figure 6. Prolonged dynasore application failed to suppress spontaneous neurotransmission. $A$, Experimental protocol. mIPSC was measured up to $1 \mathrm{~h}$ in the presence of $80 \mu \mathrm{m}$ dynasore. $\boldsymbol{B}$, Sample traces at different time points in the presence of $80 \mu \mathrm{M}$ dynasore. $\boldsymbol{C}$, Plot depicts the number of mIPSCs detected per $30 \mathrm{~s}$ period during 1 -h-long recordings. $\boldsymbol{D}$, The amplitude of $\mathrm{mIPSCS}$ show an increasing trend that did not reach significance $(p=0.056 ; n=6)$. , Comparison of the amplitudes of elPSCs detected before and after $1 \mathrm{~h}$ incubation in $80 \mu \mathrm{m}(n=4)$. The $1 \mathrm{~h}$ incubation in dynasore at rest left $40 \%$ of elPSCs intact. The decay time constant of elPSCs before and after prolonged dynasore application were not altered $(n=4 ; p>0.4)$.
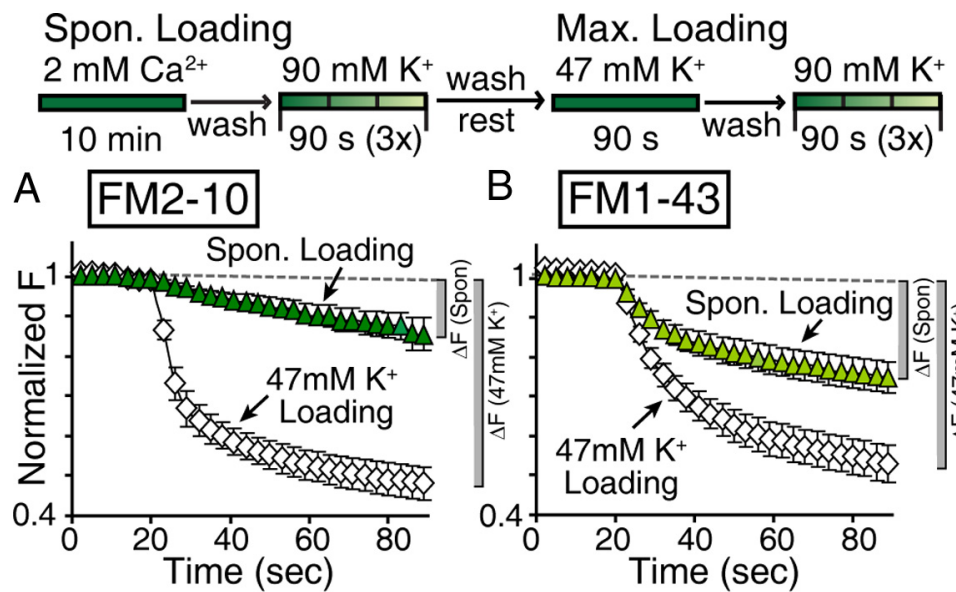

B FM1-43
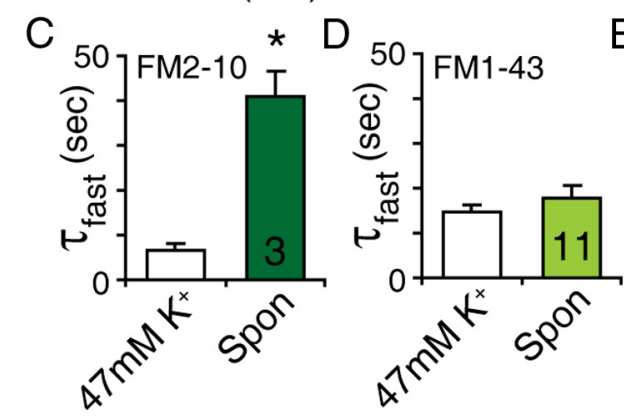

$\mathrm{E}$

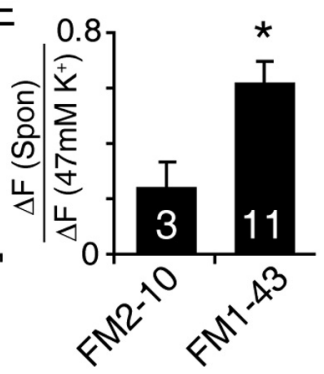

Figure 7. Properties of spontaneous synaptic vesicle trafficking reported by FM2-10 and FM1-43 diverge. The top outlines the experimental paradigm used to label vesicles that recycle spontaneously or in response to activity. $\boldsymbol{A}$, When FM2-10 was used to label vesicle at rest or during depolarization, the kinetics of subsequent dye loss during $90 \mathrm{~mm} \mathrm{~K}^{+}$stimulation was different depending on the loading protocol. Vesicles labeled with spontaneous dye uptake were refractory to $90 \mathrm{~mm} \mathrm{~K}^{+}$stimulation, leading to slow release kinetics. $\boldsymbol{B}$, In contrast, uptake of FM1-43 at rest or in response to stimulation resulted in fast dye loss regardless of the mode of dye uptake. $\boldsymbol{C}$, Bar graph depicts the average time constants of dye loss after FM2-10 uptake during activity and at rest $(n=3,520$ synapses for both conditions; $p<0.01) .{ }^{*} p<0.05$. D, Bar graph depicts the average time constants of dye loss after FM1-43 uptake during activity and at rest $(n=11,1760$ synapses for both conditions; $p>0.2)$. $\boldsymbol{E}$, The fraction of vesicles that take up FM1-43 spontaneously is larger than those labeled by FM2-10 at rest with respect to maximal dye uptake induced by $47 \mathrm{~mm} \mathrm{~K}^{+}$stimulation $\left[\Delta F\left(\right.\right.$ Spontaneous loading protocol) $\left./ \Delta F\left(47 \mathrm{~mm} \mathrm{~K}^{+}\right)\right]\left({ }^{*} p<0.05\right)$. al., 2005), whereas synapses that took up FM1-43 under resting conditions showed robust dye loss during $90 \mathrm{~mm} \mathrm{~K}^{+}$stimulation, as reported by Groemer and Klingauf (2007) (Fig. $7 B, D$ ). To examine whether FM1-43 and FM2-10 labeled the same fraction of vesicles that recycle spontaneously, we quantified the ratio of the amount of spontaneous dye uptake $[\Delta \mathrm{F}$ (Spon)] to the amount of dye taken up in response to $47 \mathrm{mM} \mathrm{K}^{+}$depolarization $[\Delta \mathrm{F}$ $\left.\left(47 \mathrm{mM} \mathrm{K}^{+}\right)\right]$(Fig. 7E). In the case of FM210 , the ratio was $\sim 24 \%$, whereas spontaneous uptake of FM1-43 labeled a significantly larger fraction of vesicles approaching $62 \%$ (Fig. 7E). This finding suggests that the number of vesicles labeled by FM2-10 and FM1-43 under resting conditions shows only partial overlap.

To verify the premise that FM2-10 uptake accurately reports the properties of spontaneous synaptic vesicle trafficking, we compared its behavior with the red shifted structurally similar styryl dye FM5-95 $(8 \mu \mathrm{M})$. For this purpose, we acquired destaining profiles of the two dyes simultaneously after spontaneous or stimulation-dependent uptake. To optimize image acquisition settings, we obtained spectral signature of each dye when nerve terminals were labeled with either dye alone or both dyes together. Figure $8 \mathrm{~A}$ depicts the spectral signature of FM2-10 when excited at $488 \mathrm{~nm}$ and FM5-95 after excitation at $543 \mathrm{~nm}$. The emission peak of FM2-10 was at $570 \mathrm{~nm}$ with a second peak at $528 \mathrm{~nm}$, whereas FM5-95 showed a strong emission peak near $660 \mathrm{~nm}$ (Fig. 8A). The emission peaks of both dyes did not show a significant shift when synapses were labeled with both dyes at the same time. To compare the mobilization kinetics of vesicles recycling spontaneously or in response to activity, we used a dye-labeling paradigm and image-acquisition setting akin to previous work by Groemer and Klingauf (2007) (Fig. 8). To label activity-dependent recycling vesicles, we used 90 -s-long $47 \mathrm{~mm} \mathrm{~K}^{+}$ mediated depolarization in the presence of FM2-10 (green) or FM5-95 (red), and, after 5 min perfusion with dye-free solution, we exposed neurons to the FM dye with the other color for $10 \mathrm{~min}$ in the presence of 1 $\mu \mathrm{M}$ TTX to label spontaneously recycling synaptic vesicles. Once excess dye was washed out $(\sim 10 \mathrm{~min})$, we monitored dye destaining profiles in both green and red emission windows simultaneously during three consecutive rounds of $90 \mathrm{~mm} \mathrm{~K}^{+}$application (90 s each) (Fig. 8). The destaining profiles emerging from individual synapses were collected at maximum emission windows for each dye (FM2-10, 500-530 nm; 
FM5-95, 650-710 nm) (Fig. 8B), and their kinetics were fitted to a double-exponential decay function with fast and slow time constants $\left(\tau_{\text {fast }}\right.$ and $\left.\tau_{\text {slow }}\right)$. When both FM dyes were taken up in response to $47 \mathrm{~mm} \mathrm{~K}^{+}$depolarization, $\tau_{\text {fast }}$ of FM2-10 and FM5-95 was mostly comparable (Fig. $8 D, G$ ). However, when we used FM2-10 to label spontaneously recycling vesicles and FM5-95 to label synaptic vesicles that recycle in response to stimulation, $\tau_{\text {fast }}$ of FM2-10 was slower than $\tau_{\text {fast }}$ of FM5-95 (Fig. 8E,H). When we exchanged the dyes and used FM5-95 labeling for spontaneously recycling vesicle pool, $\tau_{\text {fast }}$ of FM5-95 became slower compared with $\tau_{\text {fast }}$ of FM2-10 (Fig. $8 F, I)$. A particular advantage of these experiments stem from the fact the divergent destaining profiles were obtained simultaneously from the same synaptic bouton. Therefore, we further compared the destaining kinetics of the two dyes in a pairwise manner using the ratio of their fast destaining time constants $\left(\tau_{\text {fast }}\right)$ at each synapse. The average ratio of these fast time constants ( $\tau_{\text {fast }}$ of FM2-10/ $\tau_{\text {fast }}$ of FM5-95) when both dyes were taken up in response to $47 \mathrm{~mm} \mathrm{~K}^{+}$depolarization was $\sim 1.04$ (Fig. $8 J, K$ ). In agreement with the premise that spontaneous and stimulation-dependent dye uptake selectively labels kinetically separable vesicle pools, this ratio was increased to $\sim 2.81$ when FM2-10 was used to label vesicles recycling spontaneously and decreased to $\sim 0.52$ when FM5-95 was used to mark spontaneous synaptic vesicle recycling (Fig. 8J,K). This dichotomy between spontaneous versus evoked uptake of styryl dyes was also detectable when we compared the behavior of FM1-43 to FM5-95 using spectral imaging (supplemental Fig. 1, available at www.jneurosci. org as supplemental material).

\section{Differential uptake of FM1-43 and} FM2-10 reveals two parallel endocytic processes at rest

Why do the dyes FM1-43 and FM2-10 (or FM5-95) display divergent destaining kinetics after spontaneous uptake? These two dyes differ with respect to their relative hydrophobicity (Klingauf et al., 1998; Gaffield and Betz, 2006), and previous studies have suggested that certain slow forms of endocytosis that follow strong bouts of neuronal activity may selectively trap more hydrophobic FM1-43 but not FM2-10 (Richards et al., 2000, 2003; Evans and Cousin, 2007). This is presumably because in dye-free solution, in contrast to FM1-43, FM2-10 can be more swiftly removed from the endocytic struc-
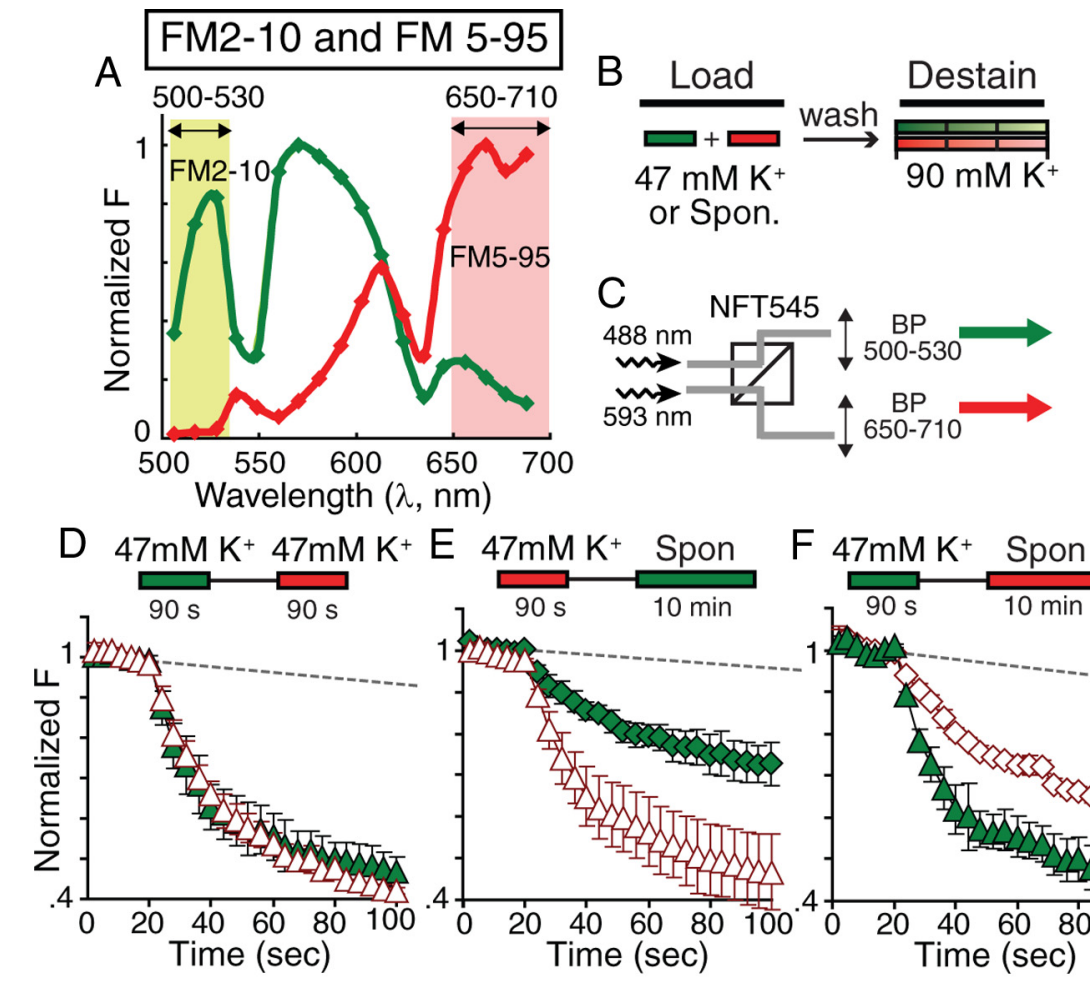

FM5-95
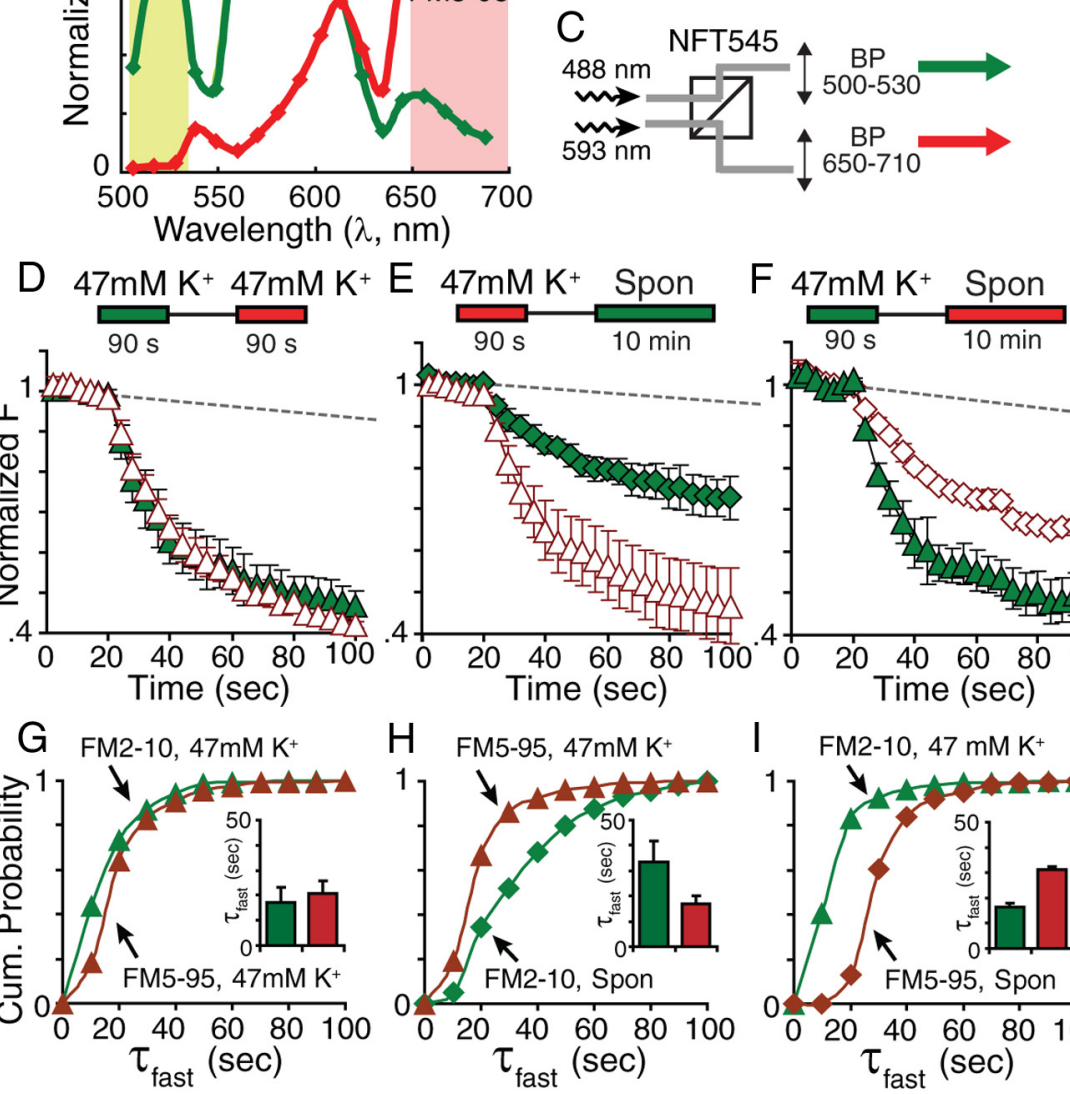

H FM5-95, 47mM K+ I
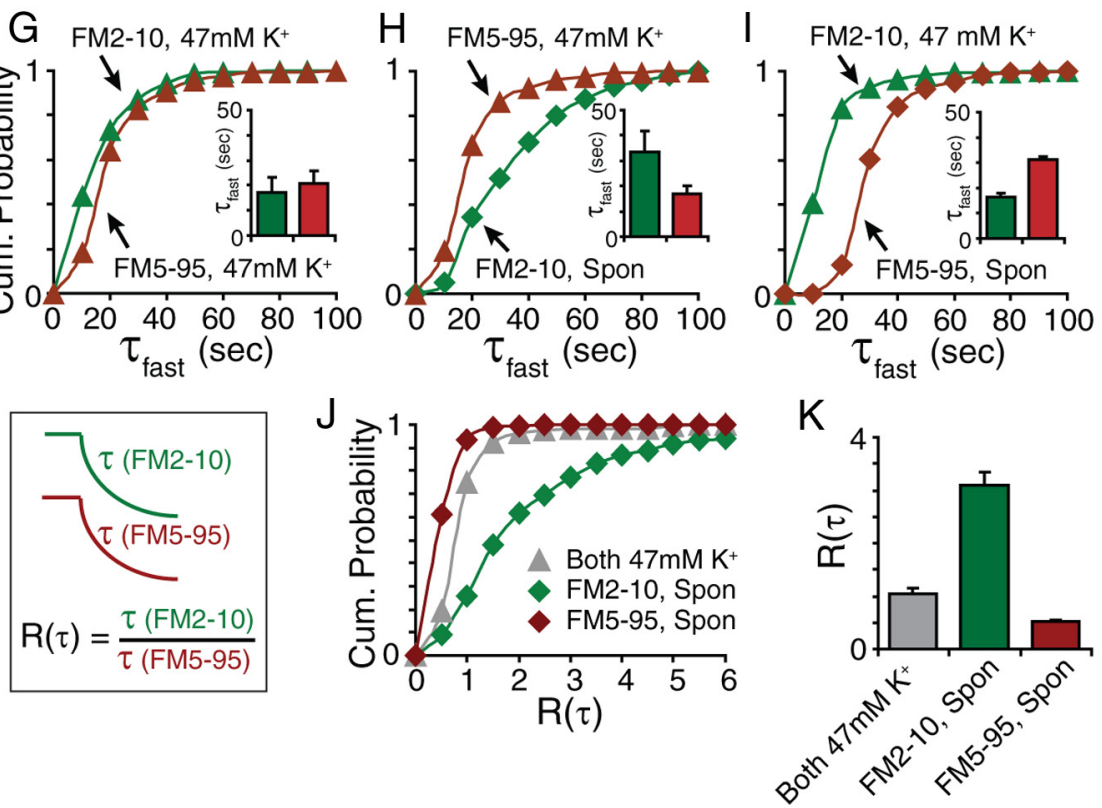

Figure 8. Simultaneous monitoring of FM2-10 and FM5-95 release shows that spontaneously trafficking vesicles are refractory to activity. $\boldsymbol{A}$, Emission spectra of FM2-10 and FM5-95 during 488 and $543 \mathrm{~nm}$ excitation. $\boldsymbol{B}$, Experimental paradigm depicting dual-color confocal experiments with FM2-10 and FM5-95. C, Illustration of confocal configuration for dual-color experiments. Samples were excited at both 488 and $543 \mathrm{~nm}$. For green signals, the emission passed through the bandpass (BP) filter $500-530$ $\mathrm{nm}$ was collected. For red signals, the fluorescence emission between 650 and $710 \mathrm{~nm}$ was collected. $\boldsymbol{D}-\boldsymbol{F}$, The graphs depict the average FM dye destaining kinetics from all experiments $(n=3-4,330-470$ synapses under each condition). The dye loading condition for each panel is illustrated at the top. FM2-10 and FM5-95 behave in a very similar manner when loaded during depolarization, presumably attributable to their similar hydrophobicities (stemming from the lengths of their hydrocarbon tails). After spontaneous uptake, however, FM2-10 and FM5-95 exhibited slower dye loss in response to depolarization (FM2-10 in $E$ and FM5-95 in F). G-I, Analysis of the fast time constants of destaining ( $\left.\tau_{\text {fast }}\right)$ for FM2-10 and FM5-95 at a individual synapses. G, When both dyes were used for activity-dependent labeling, $\tau_{\text {fast }}$ of FM2-10 and of FM5-95 was comparable (330 synapses from 3 experiments; $p>0.6)$ as well as the total amount of dye uptake $(\Delta F)$, and the fraction labeled spontaneously $[\Delta F(\%)](p>0.2)$. $\boldsymbol{H}$, When FM2-10 was used for spontaneous labeling, $\tau_{\text {fast }}$ of FM2-10 was slower than that of FM5-95 (320 synapses from 4 experiments; $p<0.05$ ).I, When FM5-95 was used for spontaneous labeling, $\tau_{\text {fast }}$ of FM5-95 was slower than that of FM2-10 (340 synapses from 4 experiments; $p<0.01)$. $\boldsymbol{J}, \boldsymbol{K}$, Analysis of the ratio of $\tau_{\text {fast }}[R(\tau)]$ measurements at individual synapses. $R(\tau)$ was defined as $\tau_{\text {fast }}\left(\right.$ FM2-10) $/ \tau_{\text {fast }}($ FM5-95). The distribution as well as the mean $R(\tau)$ values were different depending on the manner in which dyes were taken up $(p<0.001)$. 


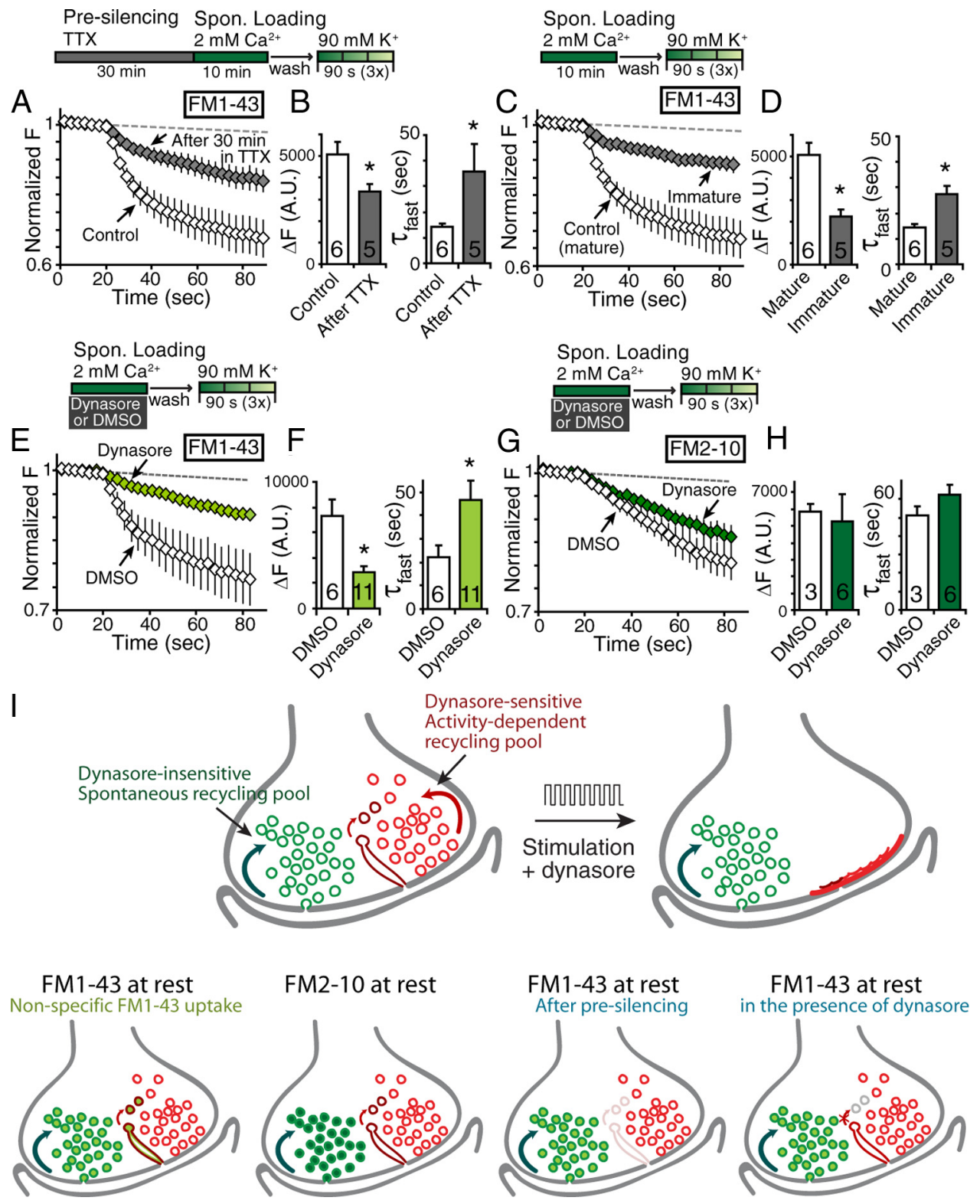

Figure 9. FM1-43-labeled synaptic vesicles at rest reflects the history of activity in a given set of synapses. $A, B$, Inhibition of neuronal network activity for 30 min in TTX reduced the extent of FM1-43 uptake at rest $(p<0.05)$ and slowed the rate of subsequent dye loss during depolarization $(p<0.05 ; 1025$ synapses from 6 experiments for control, and 950 synapses from 5 experiments for TTX-treated group) ${ }^{*} p<0.05$. C, $\boldsymbol{D}$, In immature synapses (10-12 DIV), FM1-43 labeled a smaller pool of synaptic vesicles at rest that were mobilized with a relatively slow rate during $90 \mathrm{~mm}$ $\mathrm{K}^{+}$application ( $p<0.001 ; 1025$ mature synapses from 6 experiments, 420 immature synapses from 5 experiments). ${ }^{*} p<0.05 . \boldsymbol{E}$, $\boldsymbol{F}$, The presence of $80 \mu \mathrm{m}$ dynasore caused a significant decrease in the extent of spontaneous FM1-43 uptake at rest ( $p<0.001)$ and decreased the $\tau_{\text {fast }}$ of dye destaining significantly $(p<0.05 ; 490$ synapses from 6 experiments for DMSO, 1000 synapses from 11 experiments for dynasore). ${ }^{*} p<0.05$. $\mathbf{G}, \boldsymbol{H}$, However, in the case of FM2-10, dynasore application failed to alter the extent of spontaneous dye uptake or subsequent dye mobilization ( $p>0.2$; 280 synapses from 3 experiments for DMSO, 550 synapses from 6 experiments for dynasore).I, Top, The diagram depicts two synaptic vesicle pools that coexist within individual synaptic boutons and selectively maintain spontaneous or evoked neurotransmission in parallel. Activity-dependent stimulation in the presence of dynasore leads to depletion of activity-dependent recycling pool but leaves spontaneous recycling intact. Bottom, Under resting conditions that follow activity spontaneous uptake and release of FM2-10 is a selective reporter for genuine spontaneous synaptic vesicle trafficking, which in turn gives rise to spontaneous neurotransmission. FM1-43, conversely, labels two endocytic processes: one that overlaps with spontaneous synaptic vesicle trafficking, and the other an asynchronous form of endocytosis that follows strong bouts of activity with a delay and requires dynamin function. After long periods of rest, this asynchronous endocytosis ceases and FM2-10 as well as FM1-43 labels the same spontaneously recycling pool. Vesicle recycling via this asynchronous form of endocytosis can also be inhibited by dynasore.

tures formed after strong activity. Therefore, in the next set of experiments, we tested whether the release kinetics of FM1-43 after spontaneous uptake is sensitive to the previous background network activity levels present in the hippocampal cultures (Virmani et al., 2006). To alter the level of background network activity, we preincubated mature cultures with $1 \mu \mathrm{M}$ TTX for 30 min ("pre-silencing") before dye uptake and release (Fig. 9A,B) or we used immature hippocampal cultures, which typically have lower network activity levels (Fig. 9C,D). Under both conditions, spontaneous uptake of FM1-43 (10 min in TTX) resulted in slow dye loss during subsequent depolarization, and the size of synaptic vesicle pool labeled with FM1-43 showed a significant decrease (Fig. 9A-D). Conversely, the conditions did not significantly affect the stimulation-dependent uptake and release of FM1-43 (data not shown).

Our observations in the present study suggest that acute inhibition of dynamin function with dynasore selectively suppresses evoked but not spontaneous synaptic vesicle trafficking. These 
results predict that FM1-43 uptake under resting conditions should be sensitive to dynasore as the degree of spontaneous FM1-43 labeling is sensitive to previous activity. To test this prediction, we exposed cells to FM1-43 in the presence of $80 \mu \mathrm{M}$ dynasore or $1 \%$ DMSO for 10 min under resting conditions (in TTX without pre-silencing) and subsequently stimulated with 90 $\mathrm{mM} \mathrm{K}^{+}$after dye removal (Fig. 9E,F). We found that dynasore application indeed decreased the extent of spontaneous FM1-43 uptake and slowed its release kinetics in response to $90 \mathrm{mM} \mathrm{K}^{+}$ challenge (Fig. 9E,F). This effect of dynasore was specific to FM1-43 as spontaneous trafficking of FM2-10 was essentially unaffected by the presence of dynasore during dye uptake (Fig. $9 G, H)$. Together, these results suggest that, under standard conditions, spontaneous uptake and release of FM2-10 is a selective reporter for genuine spontaneous synaptic vesicle trafficking, which gives rise to spontaneous neurotransmission. FM1-43, conversely, labels two endocytic processes: one that overlaps with spontaneous synaptic vesicle trafficking and the other an asynchronous form of endocytosis that follows bouts of activity with a delay and requires dynamin function (Fig. 9I).

Why does 10-min-long incubation in FM2-10 fail to label this slow activity-dependent form of endocytosis? Findings from a previous study, in which we had examined differential uptake of FM1-43 versus FM2-10 after strong depolarization of hippocampal cultures, may address this question (Virmani et al., 2003). In this study, under normal conditions, we detected negligible additional uptake of FM2-10 after strong stimulation when dye washout was delayed for $10 \mathrm{~min}$. However, we could alter this situation by overexpressing a splice variant of synaptotagmin-7, which promoted a form of endocytosis reminiscent of delayed retrieval seen in other systems (Evans and Cousin, 2007). Even under these circumstances, dye labeling after washout of FM2-10 with a 10 min delay did not reach the fraction labeled with FM143 , suggesting that, in hippocampal synapses, removal of FM2-10 with a 10 min delay is not sufficient to tag this slow endocytosis that persists after sustained activity.

\section{Discussion}

In the present study, we made several key observations on how dynamin-mediated synaptic vesicle endocytosis contributes to neurotransmission by taking advantage of dynasore, a reversible small molecule inhibitor of dynamin function. First, in agreement with previous reports, we verified that dynasore is a bona fide and potent inhibitor of the GTPase activity of dynamin (Macia et al., 2006; Kirchhausen et al., 2008). This inhibition of dynamin function, in turn, was correlated with a marked suppression of synaptic vesicle endocytosis during and after synaptic stimulation. Second, dynasore, at concentrations that potently inhibits dynamin activation as well as synaptic vesicle endocytosis, caused use-dependent depression of evoked synaptic transmission at an extremely rapid timescale $(\sim 1 \mathrm{~s})$. This finding indicates that neurotransmission during repetitive stimulation requires rapid reuse of synaptic vesicles endocytosed in a dynamin-dependent manner (Pyle et al., 2000; Sara et al., 2002; Harata et al., 2006; Ertunc et al., 2007). Third, under the same settings, acute inhibition of dynamin affects synchronous as well as asynchronous release, suggesting that both forms of release relies on dynamindependent synaptic vesicle recycling. Finally, the experiments we present here suggest that spontaneous neurotransmission is consistently resilient to acute inhibition of dynamin. In contrast to the strong impact of acute dynamin inhibition on evoked synaptic vesicle recycling, dynasore treatment did not significantly affect spontaneous neurotransmission and caused partial de- crease in hypertonic sucrose-triggered release. Once synaptic vesicles responsive to $\mathrm{Ca}^{2+}$-evoked release were depleted via elevated potassium stimulation in the presence of dynasore, hypertonicity-induced release showed a marked reduction ( $\sim 40 \%$ of control), but spontaneous release was left essentially intact. Because the magnitude and duration of high potassium stimulation used in these experiments is sufficient to mobilize nearly all actively recycling vesicles in a synapse (Klingauf et al., 1998; Kavalali et al., 1999a; Sara et al., 2005; Groemer and Klingauf, 2007), it is rather unexpected that at least $40 \%$ of the vesicle pool mobilized by hypertonic sucrose and almost all spontaneous neurotransmission was left intact by this maneuver. These findings contrast the previous observations that both hypertonic sucrose-driven release and spontaneous neurotransmission rely on synaptic vesicle reuse because they can be swiftly suppressed in response to application of vacuolar ATPase inhibitors such as folimycin (Ertunc et al., 2007). This dichotomy suggests that these vesicles recycle, albeit via a mechanism that is not susceptible to acute dynamin inhibition. Together, our results can be reconciled with a model in which spontaneous release is sustained by a vesicle pool, which reluctantly responds to activity and partly overlaps with the pool that fuses in response to hypertonic stimulation. Moreover, although these vesicles can be recruited to fuse during strong stimulation, they do not require dynamin activation for recycling. This proposal is consistent with recent studies in dorsal root ganglion neurons and in the calyx of Held that have shown that neurotransmission can be partly maintained by a pool of vesicles recycling in a dynaminindependent manner (Zhang et al., 2004; Xu et al., 2008). Furthermore, spontaneous neurotransmission can be maintained after cholesterol depletion, which selectively impairs evoked release as well as clathrin-mediated endocytosis (Subtil et al., 1999; Zamir and Charlton, 2006; Wasser et al., 2007). Interestingly, cholesterol depletion causes a substantial increase in the rate of spontaneous transmission, although most vesicles that perform evoked neurotransmission are either depleted or impaired to fuse (Zamir and Charlton, 2006; Wasser et al., 2007). This finding is reminiscent of the increased spontaneous transmission we detected here after prolonged dynamin inhibition at rest (Fig. 6). The reciprocal interaction between the two forms of neurotransmission may suggest that, once activity-dependent synaptic vesicle recycling is impaired, a secondary pool of vesicles becomes unhindered and augments spontaneous release. This premise agrees well with a recent study at the calyx of Held in which, after depletion of dynamin-dependent vesicle recycling, an auxiliary vesicle pool was recruited to maintain release independent of GTP hydrolysis (Xu et al., 2008). This pool may overlap with the resting pool of vesicles and substantially contribute to spontaneous neurotransmitter release (Fredj and Burrone, 2009).

Furthermore, in this study, we took advantage of the differential dynamin dependence of spontaneous and evoked forms of synaptic vesicle trafficking and gained new insight into previous contradictory proposals on whether the two forms release originate from the same set of vesicles (Sara et al., 2005; Groemer and Klingauf, 2007). We could replicate the original finding that release kinetics of styryl dye FM2-10 was extremely sensitive to its mode of uptake into nerve terminals. After stimulation-triggered dye retrieval, FM2-10-labeled vesicles showed rapid destaining, but after spontaneous dye uptake, their fluorescence loss was refractory to strong depolarization. In contrast, FM1-43 release showed robust destaining component regardless of its mode of uptake. We could also verify these observations by monitoring release of a pair of green (FM2-10) and red (FM5-95) dyes simul- 
taneously after their spontaneous or activity-dependent uptake and found that their release kinetics depend heavily on the way they were retrieved. Subsequent experiments showed that the discrepancy between FM2-10 and FM1-43 originated from selective dynasore-sensitive uptake of FM1-43 by a slow asynchronous form of endocytosis that followed strong bouts of network activity present in these dissociated cultures (Fig. 9I). This result is consistent with previous reports that certain forms of endocytosis triggered after sustained neuronal activity selectively traps more hydrophobic FM1-43 but not FM2-10 (Richards et al., 2000, 2003; Virmani et al., 2003; Evans and Cousin, 2007), presumably because in dye-free solution FM2-10 can be rapidly removed from the endocytic structures formed after strong activity. Our findings indicate that, under resting conditions, FM2-10 uptake solely labels canonical spontaneous synaptic vesicle trafficking, which is detached from previous activity and insensitive to dynamin inhibition. These experiments also uncover a slow form of endocytosis that occurs at rest but still coupled to previous activity that selectively traps FM1-43. These results also help reconcile the difference between the previous findings from our group and those of Groemer and Klingauf (2007) by indicating that the different observations originate from reliance on distinct dye species that label only partially overlapping vesicular compartments.

The findings we present here are consistent with previous work from manipulation of dynamin function using dominantnegative or genetic knock-out approaches. Studies on the Drosophila temperature-sensitive dynamin mutant shibire have provided strong support for the hypothesis that endocytosed vesicles are reused rapidly to maintain neurotransmission during activity (Kawasaki et al., 2000). Kinetic analysis of synaptic depression in shibire flies suggests a recycling rate of one to two vesicles per second per release site (Delgado et al., 2000). In addition, a recent study on a mouse knock-out of dynamin 1 also revealed a similar rapid synaptic depression attributable to loss of vesicle endocytosis occurring during synaptic stimulation as well as inhibition of synaptophysin-pHluorin trafficking during activity (Ferguson et al., 2007).

In the model presented above, we cannot exclude the possibility that the residual dynamin activity uninhibited by dynasore or a dynasore-insensitive isoform of dynamin (perhaps dynamin 3) may still be sufficient to sustain spontaneous neurotransmission. Nevertheless, this alternative explanation would suggest that the two forms of vesicle recycling are segregated by their reliance on two markedly distinct levels of dynamin activity or different dynamin isoforms rather than their dependence on dynamin per se. In agreement with this proposal, in the Drosophila neuromuscular junction, temperature-sensitive shibire mutants show suppression of evoked as well as spontaneous transmission after strong stimulation, indicating that the two forms of transmission are dynamin dependent. However, in this system, recovery of spontaneous transmission after relief of dynamin inhibition requires repopulation of the total vesicle pool, whereas evoked release recovers once the readily releasable pool is repopulated with vesicles (Koenig and Ikeda, 1996, 1999). One caveat of the shibire system is the dominant-negative nature of the mutation, which may interfere with other trafficking pathways that may act in parallel with the dynamin-dependent trafficking pathway. This caveat of the shibire system is supported by the recently characterized phenotype of mouse deficient in dynamin 1, which shows a highly selective suppression of evoked synaptic vesicle trafficking (Ferguson et al., 2007).

In summary, the results we present here bring additional insight into the complexity of synaptic vesicle trafficking within individual nerve terminals. Overall, our findings are consistent with the hypothesis that, in central synapses, two distinct synaptic vesicle trafficking pathways operate in parallel and maintain evoked versus spontaneous forms neurotransmission, thus impacting neuronal signaling in a divergent manner (Sutton et al., 2007; Sutton and Schuman, 2009). Furthermore, spontaneous neurotransmission operates independent of canonical dynaminmediated synaptic vesicle endocytosis or may have only marginal dependence on dynamin function (Krämer and Kavalali, 2008; $\mathrm{Xu}$ et al., 2008). Future studies will need to address the molecular mechanism that underlie spontaneous synaptic vesicle endocytosis and identify the machinery required to trigger synaptic vesicle fission independent of dynamin.

\section{References}

Achiriloaie M, Barylko B, Albanesi JP (1999) Essential role of the dynamin pleckstrin homology domain in receptor-mediated endocytosis. Mol Cell Biol 19:1410-1415.

Atasoy D, Ertunc M, Moulder KL, Blackwell J, Chung C, Su J, Kavalali ET (2008) Spontaneous and evoked glutamate release activates two populations of NMDA receptors with limited overlap. J Neurosci 28:10151-10166.

Barylko B, Binns D, Lin KM, Atkinson MA, Jameson DM, Yin HL, Albanesi JP (1998) Synergistic activation of dynamin GTPase by Grb2 and phosphoinositides. J Biol Chem 273:3791-3797.

Cremona O, De Camilli P (1997) Synaptic vesicle endocytosis. Curr Opin Neurobiol 7:323-330.

Delgado R, Maureira C, Oliva C, Kidokoro Y, Labarca P (2000) Size of vesicle pools, rates of mobilization, and recycling at neuromuscular synapses of a Drosophila mutant, shibire. Neuron 28:941-953.

Dröse S, Altendorf K (1997) Bafilomycins and concanamycins as inhibitors of V-ATPases and P-ATPases. J Exp Biol 200:1-8.

Ertunc M, Sara Y, Chung C, Atasoy D, Virmani T, Kavalali ET (2007) Fast synaptic vesicle reuse slows the rate of synaptic depression in the CA1 region of hippocampus. J Neurosci 27:341-354.

Evans GJ, Cousin MA (2007) Activity-dependent control of slow synaptic vesicle endocytosis by cyclin-dependent kinase 5. J Neurosci 27:401-411.

Fatt P, Katz B (1952) Spontaneous subthreshold activity at motor nerve endings. J Physiol 117:109-128.

Ferguson SM, Brasnjo G, Hayashi M, Wölfel M, Collesi C, Giovedi S, Raimondi A, Gong LW, Ariel P, Paradise S, O’Toole E, Flavell R, Cremona O, Miesenböck G, Ryan TA, De Camilli P (2007) A selective activitydependent requirement for dynamin 1 in synaptic vesicle endocytosis. Science 316:570-574.

Fredj NB, Burrone J (2009) A resting pool of vesicles is responsible for spontaneous vesicle fusion at the synapse. Nat Neurosci 12:751-758.

Gaffield MA, Betz WJ (2006) Imaging synaptic vesicle exocytosis and endocytosis with FM dyes. Nat Protoc 1:2916-2921.

Groemer TW, Klingauf J (2007) Synaptic vesicles recycling spontaneously and during activity belong to the same vesicle pool. Nat Neurosci 10:145-147.

Hablitz JJ, Mathew SS, Pozzo-Miller L (2009) GABA vesicles at synapses: are there 2 distinct pools? Neuroscientist 15:218-224.

Harata N, Pyle JL, Aravanis AM, Mozhayeva M, Kavalali ET, Tsien RW (2001) Limited number of recycling vesicles in small CNS nerve terminals: implications for neural signaling and cell biology of vesicular cycling. Trends Neurosci 24:637-643.

Harata NC, Choi S, Pyle JL, Aravanis AM, Tsien RW (2006) Frequencydependent kinetics and prevalence of kiss-and-run and reuse at hippocampal synapses studied with novel quenching methods. Neuron 49:243-256.

Hefft S, Jonas P (2005) Asynchronous GABA release generates long-lasting inhibition at a hippocampal interneuron-principal neuron synapse. Nat Neurosci 8:1319-1328.

Higashijima T, Ferguson KM, Smigel MD, Gilman AG (1987) The effect of GTP and $\mathrm{Mg}^{2+}$ on the GTPase activity and the fluorescent properties of Go. J Biol Chem 262:757-761.

Kavalali ET, Klingauf J, Tsien RW (1999a) Properties of fast endocytosis at hippocampal synapses. Philos Trans R Soc Lond B Biol Sci 354:337-346.

Kavalali ET, Klingauf J, Tsien RW (1999b) Activity-dependent regulation of synaptic clustering in a hippocampal culture system. Proc Natl Acad Sci U S A 96:12893-12900. 
Kawasaki F, Hazen M, Ordway RW (2000) Fast synaptic fatigue in shibire mutants reveals a rapid requirement for dynamin in synaptic vesicle membrane trafficking. Nat Neurosci 3:859-860.

Kirchhausen T, Macia E, Pelish HE (2008) Use of dynasore, the small molecule inhibitor of dynamin, in the regulation of endocytosis. Methods Enzymol 438:77-93.

Klingauf J, Kavalali ET, Tsien RW (1998) Kinetics and regulation of fast endocytosis at hippocampal synapses. Nature 394:581-585.

Koenig JH, Ikeda K (1996) Synaptic vesicles have two distinct recycling pathways. J Cell Biol 135:797-808.

Koenig JH, Ikeda K (1999) Contribution of active zone subpopulation of vesicles to evoked and spontaneous release. J Neurophysiol 81:1495-1505.

Krämer H, Kavalali ET (2008) Dynamin-independent synaptic vesicle retrieval? Nat Neurosci 11:6-8.

Li Z, Burrone J, Tyler WJ, Hartman KN, Albeanu DF, Murthy VN (2005) Synaptic vesicle recycling studied in transgenic mice expressing synaptopHluorin. Proc Natl Acad Sci U S A 102:6131-6136.

Lin MY, Teng H, Wilkinson RS (2005) Vesicles in snake motor terminals comprise one functional pool and utilize a single recycling strategy at all stimulus frequencies. J Physiol 568:413-421.

Lou X, Scheuss V, Schneggenburger R (2005) Allosteric modulation of the presynaptic $\mathrm{Ca}^{2+}$ sensor for vesicle fusion. Nature 435:497-501.

Lu T, Trussell LO (2000) Inhibitory transmission mediated by asynchronous transmitter release. Neuron 26:683-694.

Macia E, Ehrlich M, Massol R, Boucrot E, Brunner C, Kirchhausen T (2006) Dynasore, a cell-permeable inhibitor of dynamin. Dev Cell 10:839-850.

Mathew SS, Pozzo-Miller L, Hablitz JJ (2008) Kainate modulates presynaptic GABA release from two vesicle pools. J Neurosci 28:725-731.

Maximov A, Südhof TC (2005) Autonomous function of synaptotagmin 1 in triggering synchronous release independent of asynchronous release. Neuron 48:547-554.

Moulder KL, Mennerick S (2005) Reluctant vesicles contribute to the total readily releasable pool in glutamatergic hippocampal neurons. J Neurosci 25:3842-3850.

Moulder KL, Jiang X, Taylor AA, Shin W, Gillis KD, Mennerick S (2007) Vesicle pool heterogeneity at hippocampal glutamate and GABA synapses. J Neurosci 27:9846-9854.

Mozhayeva MG, Sara Y, Liu X, Kavalali ET (2002) Development of vesicle pools during maturation of hippocampal synapses. J Neurosci 22:654-665.

Neher E, Sakaba T (2008) Multiple roles of calcium ions in the regulation of neurotransmitter release. Neuron 59:861-872.

Newton AJ, Kirchhausen T, Murthy VN (2006) Inhibition of dynamin completely blocks compensatory synaptic vesicle endocytosis. Proc Natl Acad Sci U S A 103:17955-17960.

Otsu Y, Murphy TH (2004) Optical postsynaptic measurement of vesicle release rates for hippocampal synapses undergoing asynchronous release during train stimulation. J Neurosci 24:9076-9086.

Otsu Y, Shahrezaei V, Li B, Raymond LA, Delaney KR, Murphy TH (2004) Competition between phasic and asynchronous release for recovered synaptic vesicles at developing hippocampal autaptic synapses. J Neurosci 24:420-433.

Piedras-Rentería ES, Pyle JL, Diehn M, Glickfeld LL, Harata NC, Cao Y, Kavalali ET, Brown PO, Tsien RW (2004) Presynaptic homeostasis at CNS nerve terminals compensates for lack of a key $\mathrm{Ca}^{2+}$ entry pathway. Proc Natl Acad Sci U S A 101:3609-3614.
Praefcke GJ, McMahon HT (2004) The dynamin superfamily: universal membrane tubulation and fission molecules? Nat Rev Mol Cell Biol 5:133-147.

Pyle JL, Kavalali ET, Piedras-Rentería ES, Tsien RW (2000) Rapid reuse of readily releasable pool vesicles at hippocampal synapses. Neuron 28: 221-231.

Richards DA, Guatimosim C, Betz WJ (2000) Two endocytic recycling routes selectively fill two vesicle pools in frog motor nerve terminals. Neuron 27:551-559.

Richards DA, Guatimosim C, Rizzoli SO, Betz WJ (2003) Synaptic vesicle pools at the frog neuromuscular junction. Neuron 39:529-541.

Sankaranarayanan S, Ryan TA (2001) Calcium accelerates endocytosis of vSNAREs at hippocampal synapses. Nat Neurosci 4:129-136.

Sara Y, Mozhayeva MG, Liu X, Kavalali ET (2002) Fast vesicle recycling supports neurotransmission during sustained stimulation at hippocampal synapses. J Neurosci 22:1608-1617.

Sara Y, Virmani T, Deák F, Liu X, Kavalali ET (2005) An isolated pool of vesicles recycles at rest and drives spontaneous neurotransmission. Neuron 45:563-573.

Subtil A, Gaidarov I, Kobylarz K, Lampson MA, Keen JH, McGraw TE (1999) Acute cholesterol depletion inhibits clathrin-coated pit budding. Proc Natl Acad Sci U S A 96:6775-6780.

Sun J, Pang ZP, Qin D, Fahim AT, Adachi R, Südhof TC (2007) A dual$\mathrm{Ca}^{2+}$-sensor model for neurotransmitter release in a central synapse. Nature 450:676-682.

Sutton MA, Schuman EM (2009) Partitioning the synaptic landscape: distinct microdomains for spontaneous and spike-triggered neurotransmission. Sci Signal 2:pe19.

Sutton MA, Taylor AM, Ito HT, Pham A, Schuman EM (2007) Postsynaptic decoding of neural activity: eEF2 as a biochemical sensor coupling miniature synaptic transmission to local protein synthesis. Neuron 55:648661.

Takei K, Yoshida Y, Yamada H (2005) Regulatory mechanisms of dynamindependent endocytosis. J Biochem 137:243-247.

Virmani T, Han W, Liu X, Südhof TC, Kavalali ET (2003) Synaptotagmin 7 splice variants differentially regulate synaptic vesicle recycling. EMBO J 22:5347-5357.

Virmani T, Atasoy D, Kavalali ET (2006) Synaptic vesicle recycling adapts to chronic changes in activity. J Neurosci 26:2197-2206.

Wasser CR, Kavalali ET (2009) Leaky synapses: regulation of spontaneous neurotransmission in central synapses. Neuroscience 158:177-188.

Wasser CR, Ertunc M, Liu X, Kavalali ET (2007) Cholesterol-dependent balance between evoked and spontaneous synaptic vesicle recycling. J Physiol 579:413-429.

Xu J, McNeil B, Wu W, Nees D, Bai L, Wu LG (2008) GTP-independent rapid and slow endocytosis at a central synapse. Nat Neurosci 11:45-53.

Xu J, Pang ZP, Shin OH, Südhof TC (2009) Synaptotagmin-1 functions as a $\mathrm{Ca}^{2+}$ sensor for spontaneous release. Nat Neurosci 12:759-766.

Zamir O, Charlton MP (2006) Cholesterol and synaptic transmitter release at crayfish neuromuscular junctions. J Physiol 571:83-99.

Zhang C, Xiong W, Zheng H, Wang L, Lu B, Zhou Z (2004) Calcium- and dynamin-independent endocytosis in dorsal root ganglion neurons. Neuron 42:225-236. 